\title{
Multiquark Hadrons - A New Facet of QCD
}

\author{
Ahmed Ali \\ DESY, Hamburg \\ Nov. 9-14, 2015 \\ Regional Meeting, Islamabad
}


- Experimental Evidence for Multiquark states $X, Y, Z$

- Models for X, Y,Z Mesons

- Phenomenology of the diquark model of Tetraquarks

- The LHCb Pentaquarks $\mathbb{P}^{ \pm}(4380)$ and $\mathbb{P}^{ \pm}(4450)$

- Theoretical interpretations of the Pentaquarks

- Summary 


\section{$X(3872)$ - the poster Child of the $X, Y, Z$ Mesons}

\section{Observation of a Narrow Charmoniumlike State in Exclusive $B^{ \pm} \rightarrow K^{ \pm} \pi^{+} \pi^{-} J / \psi$ Decays}

S. -K. Choi, 5 S. L Olsen, ${ }^{6} \mathrm{~K}$. Abe, ${ }^{7}$ T. Abe, ${ }^{7}$ I Adachi, ${ }^{7}$ Byoung Sup Ahn, ${ }^{14} \mathrm{H}$. Aihara, ${ }^{43} \mathrm{~K}$ Akai, ${ }^{7}$ M. Akatsu, ${ }^{20}$ M. Akemoto ${ }^{7}{ }^{\text {Y. Asano, }},{ }^{48} \mathrm{~T}$. Aso,${ }^{47}$ V. Aulchenko, ${ }^{1}$ T. Aushev, ${ }^{11}$ A. M. Bakich, ${ }^{38} \mathrm{Y}$. Ban, ${ }^{31} \mathrm{~S}$ Banerjee, ${ }^{30}$ A. Bondar, ${ }^{1}$ A. Bozek, ${ }^{25}$ M. Bračko, ${ }^{18,12}$ J. Brodzicka, ${ }^{25}$ T. E. Browder, ${ }^{\circ}$ P. Chang, ${ }^{24}$ Y. Chao ${ }^{24} \mathrm{~K}-\mathrm{F}$. Chen, ${ }^{24}$ B. G. Cheon, ${ }^{37}$

R. Chistov, ${ }^{11}$ Y. Choi, ${ }^{37}$ Y. K. Choi, ${ }^{37}$ M. Danilov, ${ }^{11}$ L. Y. Dong, ${ }^{9}$ A. Drutskoy, ${ }^{11}$ S. Eidelman, ${ }^{1}$ V. Eiges, ${ }^{11}$ J. Flanagan, ${ }^{7}$ C. Fukunaga, ${ }^{45}$ K. Furukawa, N. Gabyshev, ${ }^{7}$ T. Gershon, B. Golob, ${ }^{77,12} \mathrm{H}$. Guler, ${ }^{6}$ R. Guo, ${ }^{22} \mathrm{C}$. Hagner, ${ }^{50} \mathrm{~F} . \mathrm{Handa},{ }^{42}$ T. Hara, ${ }^{29}$ N. C. Hastings, ${ }^{7}$ H. Hayashii, ${ }^{21}$ M. Hazumi, ${ }^{7}$ L. Hinz, ${ }^{10}$ Y. Hoshi, ${ }^{41}$ W.-S. Hou, ${ }^{24}$ Y. B. Hsiung, ${ }^{24,7}$

$\mathrm{H}$-C. Huang, ${ }^{24} \mathrm{~T}$. Iiji ma, ${ }^{20} \mathrm{~K}$. Inami, ${ }^{20} \mathrm{~A}$ Ishikawa, ${ }^{20} \mathrm{R}$. Itoh, ${ }^{7} \mathrm{M}$. Iwasaki, ${ }^{43}$ Y. Iwasaki, ${ }^{7} \mathrm{~J} . \mathrm{H}$ Kang,${ }^{52} \mathrm{~S}$. U. Kataoka, ${ }^{21}$ N. Katayama, ${ }^{7}$ H. Kawai, ${ }^{2}$ T. Kawasaki, ${ }^{27}$ H. Kichimi, ${ }^{7}$ E. Kikutani, ${ }^{7}$ H. J. Kim, ${ }^{52}$ Hyunwoo Kim, ${ }^{14}$ J. H Ki m, ${ }^{37}$

S. K. Kim, ${ }^{30}$ K. Kinoshita, ${ }^{3}$ H. Koiso, ${ }^{7}$ P. Koppenburg, ${ }^{7}$ S. Korpar, ${ }^{18}, 12$ P. Križan, ${ }^{17,12}$ P. Krokovny, ${ }^{1}$ S. Kumar, ${ }^{30}$

A. Kuzmin, ${ }^{1}$ J. S. Lange, ${ }^{4,33}$ G. Leder, ${ }^{10}$ S. H Lee, ${ }^{36}$ T. Lesiak, ${ }^{25}$ S. W. Lin, ${ }^{24}$ D. Liventsev, ${ }^{11}$ J. MacNaughton, ${ }^{10}$

G. Majumder, ${ }^{39}$ F Mandl, ${ }^{10}$ D. Marlow, ${ }^{32}$ T. Mat sumoto, ${ }^{45}$ S. Michizono, ${ }^{7}$ T. Mimashi, ${ }^{7}$ W. Mitaroff, ${ }^{10}$

K. Miyabayashi, ${ }^{21}$ H. Miyake, ${ }^{29}$ D. Mohapatra, ${ }^{50}$ G. R. Moloney, ${ }^{19}$ T. Nagamine, ${ }^{42}$ Y. Nagasaka ${ }^{8}$ T. Nakadaira, ${ }^{43}$ T. T. Nakamura, ${ }^{7}$ M. Nakao, ${ }^{7}$ Z Natkaniec, ${ }^{25} \mathrm{~S} . \mathrm{Nishida},{ }^{7} \mathrm{O}$. Nitoh, ${ }^{46}$ T. Nozaki, ${ }^{7} \mathrm{~S}$. Ogawa ${ }^{40}{ }^{40}$. Ogawa, ${ }^{7} \mathrm{~K}$. Ohmi,

Y. Ohnishi, ${ }^{7}$ T. Ohshima, ${ }^{20} \mathrm{~N}$. Ohuchi, ${ }^{7} \mathrm{~K}$ Oide, ${ }^{7}$ T. Okabe, ${ }^{20} \mathrm{~S}$. Okuno, ${ }^{13}$ W. Ostrowicz, ${ }^{25} \mathrm{H}$. Ozaki, ${ }^{7}$ H. Palka, ${ }^{25}$

H. Park, ${ }^{13}$ N. Parslow, ${ }^{38}$ L. E Piilonen, ${ }^{50}$ H. Sagawa, ${ }^{7}$ S. Saitoh, ${ }^{7}$ Y. Sakai, ${ }^{7}$ T R. Sarangi, ${ }^{40}$ M. Satapat hy, ${ }^{40}$

A Satpathy, ${ }^{7.3}$ O. Schneider, ${ }^{16}$ A J. Schwartz, ${ }^{3}$ S. Semenov, ${ }^{11}$ K. Senyo, ${ }^{20}$ R. Seuster, ${ }^{\circ}$ M. E Sevior, ${ }^{10}$ H. Shibuya, ${ }^{40}$ T. Shidara, ${ }^{7}$ B. Shwartz, ${ }^{1}$ V. Sidorov, ${ }^{1}$ N. Soni, ${ }^{30}$ S. Stanič, ${ }^{48,7}$ M. Starič, ${ }^{12}$ A Sugiyama, ${ }^{34}$ T. Sumiyoshi, ${ }^{45}$ S. Suzuki, ${ }^{51}$ F Takasaki, K. Tamai, ${ }^{7}$ N. Tamura ${ }^{27}$ M. Tanaka, ${ }^{7}$ M. Tawada, ${ }^{7}$ G. N. Taylor, ${ }^{10}$ Y Tera moto, ${ }^{28}$ T Tomura ${ }^{43} \mathrm{~K}$. Trabels, T. Tsukamoto, ${ }^{7}$ S. Uehara, ${ }^{7}$ K. Ueno, ${ }^{24}$ Y. Unno, ${ }^{2}$ S. Uno, ${ }^{7}$ G. Varner, ${ }^{6}$ K. E Var vell, ${ }^{38}$ C. C. Wang. ${ }^{24}$ C. H Wang, ${ }^{23}$ J. G. Wang, ${ }^{50}$ Y. Wat anabe, ${ }^{4+}$ E Won, ${ }^{14}$ B. D. Yabsley, ${ }^{50}$ Y. Yamada, ${ }^{7}$ A Yamaguchi, ${ }^{42}$ Y. Yamashita,${ }^{20}$ H. Yanai, ${ }^{27}$ Heyoung Yang, ${ }^{36}$ J. Ying, ${ }^{31}$ M. Yoshida, ${ }^{7}$ C. C. Zhang, ${ }^{9}$ Z P. Zhang, ${ }^{35}$ and D. Žontar ${ }^{17,12}$
- Discovery Mode : $B \rightarrow J / \psi \pi^{+} \pi^{-} K$

$M=3872.0 \pm$ $0.6 \pm 0.5 \mathrm{MeV}$

$\Gamma<2.3 \mathrm{MeV}$

$J^{P C}=$ $1^{++}[\mathrm{LHCb}]$ [PRL110, 22201(2013)]

(Belle Collaboration)
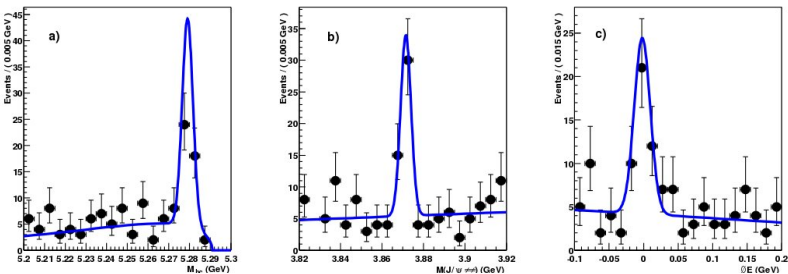
Exotica

Belle \& others [Liu et al., 13] , [Ablikim et al., 13] , [Brambilla et al., 14]

\begin{tabular}{|c|c|c|c|c|c|c|}
\hline State & $M(\mathrm{MeV})$ & $\Gamma(\mathrm{MeV})$ & $J^{P C}$ & Decay Modes & Production Modes & Also observed by \\
\hline$Y_{S}(2175)$ & $2175 \pm 8$ & $58 \pm 26$ & $1^{--}$ & $\phi f_{0}(980)$ & $\begin{array}{l}e^{+} e^{-}(\text {ISR }) \\
J / \psi \rightarrow \eta Y_{S}(2175)\end{array}$ & BaBar*, BESII \\
\hline$X(3872)$ & $3871.68 \pm 0.17$ & $<1.2$ & $1^{++}$ & $\begin{array}{l}\pi^{+} \pi^{-} J / \psi, \\
\gamma J / \psi, D D^{*}\end{array}$ & $B \rightarrow K X(3872), p \bar{p}$ & $\begin{array}{l}\text { BaBar } \\
\text { CDF, D0, BaBar, LHCb }\end{array}$ \\
\hline$Z(3900)$ & $3891.2 \pm 3.3$ & $40 \pm 8$ & $1^{+}$ & $\pi^{ \pm} J / \psi$ & $Y(4260) \rightarrow Z(3900) \pi$ & BESIII*, CLEO \\
\hline$X(3915)$ & $3914 \pm 4$ & $\begin{array}{r}28+12 \\
-14\end{array}$ & $0 / 2^{++}$ & $\omega J / \psi$ & $\gamma \gamma \rightarrow X(3915)$ & \\
\hline$Z(3930)$ & $3929 \pm 5$ & $29 \pm 10$ & $2^{++}$ & $D D$ & $\gamma \gamma \rightarrow Z(3940)$ & \\
\hline$Y(3940)$ & $3943 \pm 17$ & $87 \pm 34$ & $?++$ & $\omega J / \psi\left(\operatorname{not} D D^{*}\right)$ & $B \rightarrow K Y(3940)$ & BaBar \\
\hline$Y(4008)$ & $4008_{-49}^{+82}$ & $226_{-80}^{+97}$ & $1^{--}$ & $\pi^{+} \pi^{-} J / \psi$ & $e^{+} e^{-}$(ISR) & \\
\hline$Z(4020)$ & $4022 \pm 3$ & $8 \pm 4$ & $1^{+}$ & $\pi^{ \pm} J / \psi$ & $Y(4260) \rightarrow Z(4020) \pi$ & BESIII* (only) \\
\hline$Z(4025)$ & $4026 \pm 5$ & $25 \pm 10$ & $1^{+}$ & $\pi^{ \pm} J / \psi$ & $Y(4260) \rightarrow Z(4025) \pi$ & BESIII* (only) \\
\hline$X(4160)$ & $4156 \pm 29$ & $\begin{array}{c}139+113 \\
-65\end{array}$ & $0^{?+}$ & $D^{*} \overline{D^{*}}(\operatorname{not} D \bar{D})$ & $e^{+} e^{-} \rightarrow J / \psi X(4160)$ & \\
\hline$Y(4260)$ & $4264 \pm 12$ & $83 \pm 22$ & $1^{--}$ & $\pi^{+} \pi^{-} J / \psi$ & $e^{+} e^{-}$(ISR) & BaBar*, CLEO \\
\hline$X(4630)$ & $4634_{-11}^{+9}$ & $\begin{array}{r}92^{+41} \\
-32\end{array}$ & $1^{--}$ & $\Lambda_{c}^{+} \Lambda_{c}^{-}$ & $e^{+} e^{-}$(ISR) & \\
\hline$Y(4660)$ & $4664 \pm 12$ & $48 \pm 15$ & $1^{--}$ & $\pi^{+} \pi^{-} \psi^{\prime}$ & $e^{+} e^{-}$(ISR) & \\
\hline$Z(4050)$ & $4051_{-23}^{+24}$ & $\begin{array}{r}82+51 \\
-29\end{array}$ & $?$ & $\pi^{ \pm} \chi_{c 1}$ & $B \rightarrow K Z^{ \pm}(4050)$ & \\
\hline$Z(4250)$ & $\begin{array}{c}4248^{+}+185 \\
-45\end{array}$ & $\begin{array}{c}177^{+320} \\
-72\end{array}$ & $?$ & $\pi^{ \pm} \chi_{c 1}$ & $B \rightarrow K Z^{ \pm}(4250)$ & \\
\hline$Z(4430)$ & $4475 \pm 7$ & $\begin{array}{r}172 \pm 13^{+37} \\
-34 \\
\end{array}$ & $1^{+}$ & $\pi^{ \pm} \psi^{\prime}$ & $B \rightarrow K Z^{ \pm}(4430)$ & $\mathrm{LHCb}$ \\
\hline$Z_{b}(10610)$ & $10,607 \pm 2$ & $18.4 \pm 2.4$ & $1^{+}$ & $\pi^{ \pm} h_{b}(1,2 P), \pi^{ \pm} \mathrm{Y}(1,2,3 S)$ & $Y_{b} / Y(5 S) \rightarrow Z_{b}(10610) \pi$ & \\
\hline$Z_{b}(10650)$ & $10,652 \pm 2$ & $11.5 \pm 2.2$ & $1^{+}$ & $\pi^{ \pm} h_{b}(1,2 P), \pi^{ \pm} \mathrm{Y}(1,2,3 S)$ & $Y_{b} / Y(5 S) \rightarrow Z_{b}(10650) \pi$ & \\
\hline$Y_{b}(10890)$ & $10,890 \pm 3$ & $55 \pm 9$ & $1^{--}$ & $\pi^{+} \pi^{-} \mathrm{Y}(1,2,3 S)$ & $e^{+} e^{-} \rightarrow Y_{b}$ & \\
\hline
\end{tabular}


Exotica

Belle \& others [Liu et al., 13] , [Ablikim et al., 13] , [Brambilla et al., 14]

\begin{tabular}{|c|c|c|c|c|c|c|}
\hline State & $M(\mathrm{MeV})$ & $\Gamma(\mathrm{MeV})$ & $J^{P C}$ & Decay Modes & Production Modes & Also observed by \\
\hline$Y_{S}(2175)$ & $2175 \pm 8$ & $58 \pm 26$ & $1^{--}$ & $\phi f_{0}(980)$ & $\begin{array}{l}e^{+} e^{-}(\text {ISR }) \\
J / \psi \rightarrow \eta Y_{S}(2175)\end{array}$ & BaBar*, BESII \\
\hline$X(3872)$ & $3871.68 \pm 0.17$ & $<1.2$ & $1^{++}$ & $\begin{array}{l}\pi^{+} \pi^{-} J / \psi, \\
\gamma J / \psi, D D^{*}\end{array}$ & $B \rightarrow K X(3872), p \bar{p}$ & $\begin{array}{l}\text { BaBar } \\
\text { CDF, D0, BaBar, LHCb }\end{array}$ \\
\hline$Z(3900)$ & $3891.2 \pm 3.3$ & $40 \pm 8$ & $1^{+}$ & $\pi^{ \pm} J / \psi$ & $Y(4260) \rightarrow Z(3900) \pi$ & BESIII*, CLEO \\
\hline$X(3915)$ & $3914 \pm 4$ & $\begin{array}{r}28_{-14}^{+12} \\
-14\end{array}$ & $0 / 2^{++}$ & $\omega J / \psi$ & $\gamma \gamma \rightarrow X(3915)$ & \\
\hline$Z(3930)$ & $3929 \pm 5$ & $29 \pm 10$ & $2^{++}$ & $D D$ & $\gamma \gamma \rightarrow Z(3940)$ & \\
\hline$Y(3940)$ & $3943 \pm 17$ & $87 \pm 34$ & $?++$ & $\omega J / \psi\left(\operatorname{not} D D^{*}\right)$ & $B \rightarrow K Y(3940)$ & BaBar \\
\hline$Y(4008)$ & $4008_{-49}^{+82}$ & $226_{-80}^{+97}$ & $1^{--}$ & $\pi^{+} \pi^{-} J / \psi$ & $e^{+} e^{-}$(ISR) & \\
\hline$Z(4020)$ & $4022 \pm 3$ & $8 \pm 4$ & $1^{+}$ & $\pi^{ \pm} J / \psi$ & $Y(4260) \rightarrow Z(4020) \pi$ & BESIII* (only) \\
\hline$Z(4025)$ & $4026 \pm 5$ & $25 \pm 10$ & $1^{+}$ & $\pi^{ \pm} J / \psi$ & $Y(4260) \rightarrow Z(4025) \pi$ & BESIII* (only) \\
\hline$X(4160)$ & $4156 \pm 29$ & $\begin{array}{c}139+113 \\
-65\end{array}$ & $0^{?+}$ & $D^{*} \bar{D}^{*}(\operatorname{not} D \bar{D})$ & $e^{+} e^{-} \rightarrow J / \psi X(4160)$ & \\
\hline$Y(4260)$ & $4264 \pm 12$ & $83 \pm 22$ & $1^{--}$ & $\pi^{+} \pi^{-} J / \psi$ & $e^{+} e^{-}$(ISR) & BaBar*, CLEO \\
\hline$X(4630)$ & $4634_{-11}^{+9}$ & $\begin{array}{r}92^{+41} \\
-32\end{array}$ & $1^{--}$ & $\Lambda_{c}^{+} \Lambda_{c}^{-}$ & $e^{+} e^{-}$(ISR) & \\
\hline$Y(4660)$ & $4664 \pm 12$ & $48 \pm 15$ & $1^{--}$ & $\pi^{+} \pi^{-} \psi^{\prime}$ & $e^{+} e^{-}$(ISR) & \\
\hline$Z(4050)$ & $4051_{-23}^{+24}$ & $\begin{array}{r}82+51 \\
-29\end{array}$ & $?$ & $\pi^{ \pm} \chi_{c 1}$ & $B \rightarrow K Z^{ \pm}(4050)$ & \\
\hline$Z(4250)$ & $\begin{array}{c}4248^{+}+185 \\
-45\end{array}$ & $\begin{array}{c}177^{+320} \\
-72\end{array}$ & $?$ & $\pi^{ \pm} \chi_{c 1}$ & $B \rightarrow K Z^{ \pm}(4250)$ & \\
\hline$Z(4430)$ & $4475 \pm 7$ & $\begin{array}{r}172 \pm 13^{+37} \\
-34 \\
\end{array}$ & $1^{+}$ & $\pi^{ \pm} \psi^{\prime}$ & $B \rightarrow K Z^{ \pm}(4430)$ & $\mathrm{LHCb}$ \\
\hline$Z_{b}(10610)$ & $10,607 \pm 2$ & $18.4 \pm 2.4$ & $1^{+}$ & $\pi^{ \pm} h_{b}(1,2 P), \pi^{ \pm} \mathrm{Y}(1,2,3 S)$ & $Y_{b} / Y(5 S) \rightarrow Z_{b}(10610) \pi$ & \\
\hline$Z_{b}(10650)$ & $10,652 \pm 2$ & $11.5 \pm 2.2$ & $1^{+}$ & $\pi^{ \pm} h_{b}(1,2 P), \pi^{ \pm} \mathrm{Y}(1,2,3 S)$ & $Y_{b} / Y(5 S) \rightarrow Z_{b}(10650) \pi$ & \\
\hline$Y_{b}(10890)$ & $10,890 \pm 3$ & $55 \pm 9$ & $1^{--}$ & $\pi^{+} \pi^{-} \mathrm{Y}(1,2,3 S)$ & $e^{+} e^{-} \rightarrow Y_{b}$ & \\
\hline
\end{tabular}

\section{Light states [PDG] :}

$a_{0}(980)$ in 1965, $\sigma(600)^{\text {now }} 500$ in 1972, $f_{0}(980)$ in 1979, $\quad \kappa(980)$ in 1997 discussion reopened: ['t Hooft, Isidori, Maiani, Polosa, Riquer, PLB 08] 
Exotica

Belle \& others [Liu et al., 13] , [Ablikim et al., 13] , [Brambilla et al., 14]

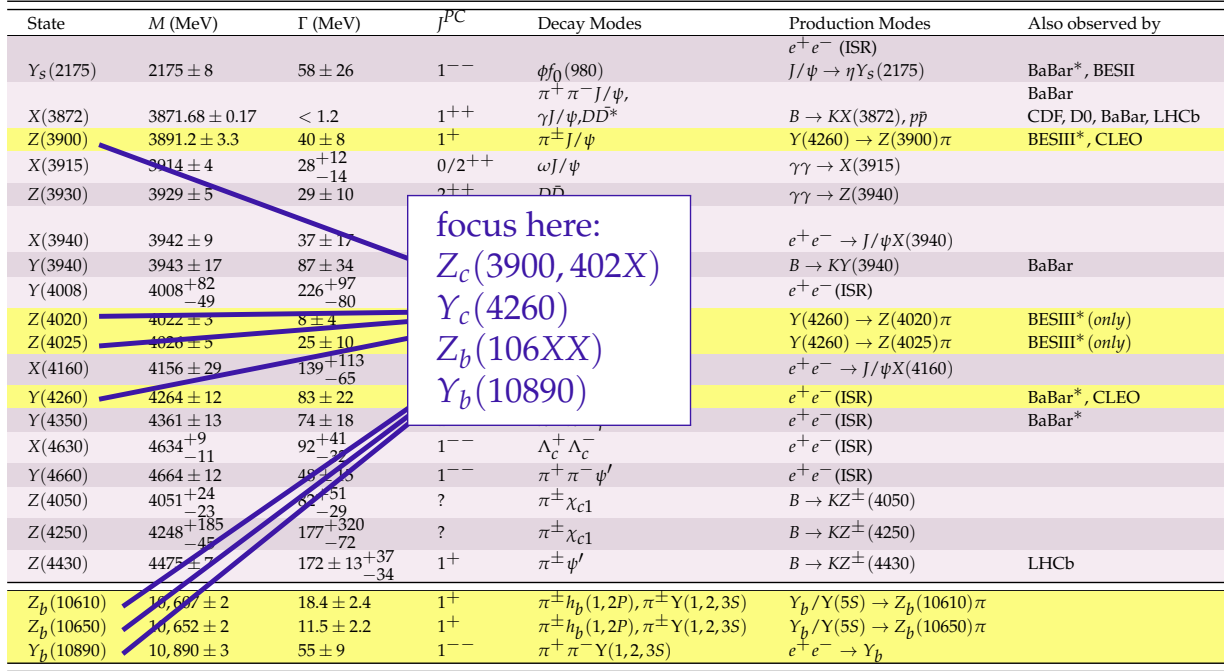

\section{Light states [PDG] :}

$a_{0}(980)$ in 1965, $\sigma(600)^{\text {now }} 500$ in 1972, $f_{0}(980)$ in 1979, $\quad \kappa(980)$ in 1997 discussion reopened: ['t Hooft, Isidori, Maiani, Polosa, Riquer, PLB 08] 


\section{Constituent Quark Model and Light States}

- Masses for light resonances in constituent model

$\rightarrow$ Flavor nonets are arranged as triangles
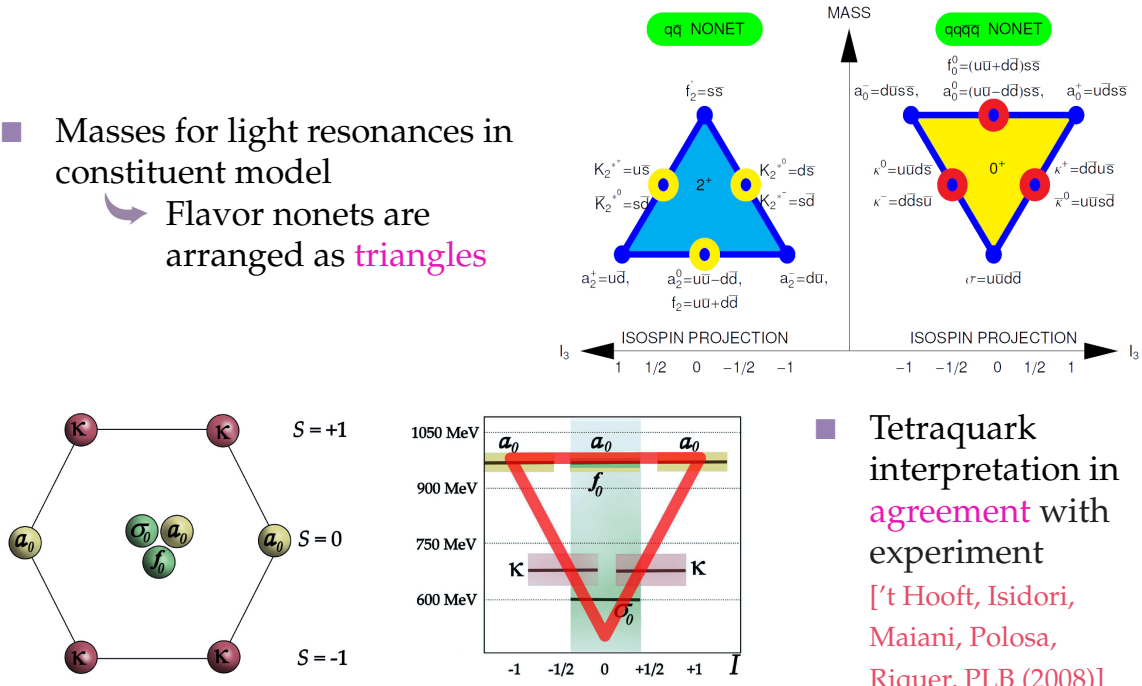

- Tetraquark interpretation in agreement with experiment ['t Hooft, Isidori, Maiani, Polosa, Riquer, PLB (2008)] 

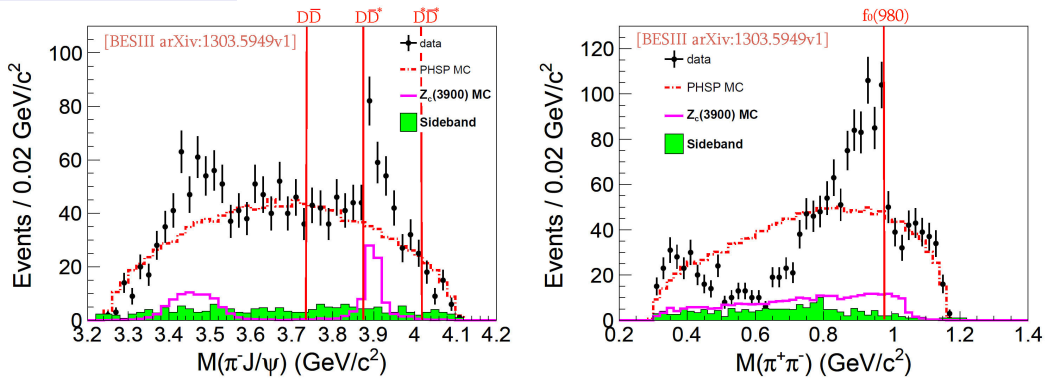

discovered in $J / \psi \pi \pi$ by BaBar, confirmed by CLEO, Belle, and BESIII

[ [BaBar, PRL 05] $: m=4252 \pm 73 \mathrm{MeV}, \Gamma=105 \pm 20 \mathrm{MeV}$

$$
\begin{aligned}
& B(J / \psi \pi \pi) \Gamma_{e+e-}=7.5 \pm 1.2 \mathrm{eV} \\
&\left.\Gamma\left(Y_{c}(4260) \rightarrow J / \psi \pi \pi\right)>0.5 \mathrm{MeV} \text { (limit on } \Gamma_{e+e-}\right) \\
& \text { (at least } \mathcal{O}(10) \text { enhanced vs charmonia) }
\end{aligned}
$$

candidates: hybrids, tetraquarks, $D_{1} \bar{D}$ molecule, $\ldots$

Dipion mass spectrum dominated by $f_{0}(980)$ - a tetraquark candidate itself 
Observation of $Z_{c}(3900)^{ \pm}$in the decay $Y(4260) \rightarrow \pi Z_{\mathcal{c}}(3900)$

K. Seth \& co. (a) 4.170 GeV

hep-ex: 1304.3036

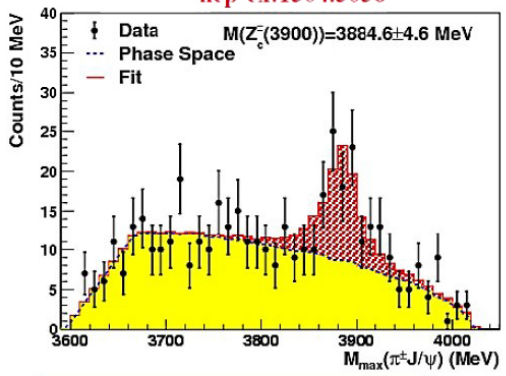

$M=(3885 \pm 5 \pm 1) M e V / c^{2}$

$\Gamma=(34 \pm 12 \pm 4) \mathrm{MeV} / \mathrm{c}^{2}$

$81 \pm 20$ events

$6.1 \sigma$
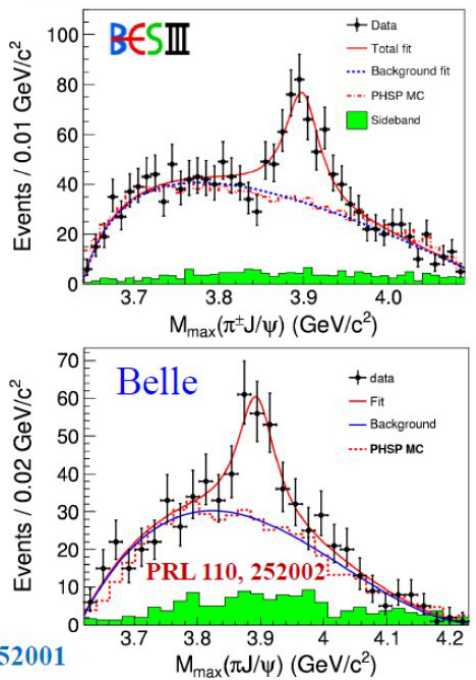
$M_{\max }(\pi \mathrm{J} / \psi)\left(\mathrm{GeV} / \mathrm{c}^{2}\right)$

PRL 110, 252001 
Summary of Charmonia and Charmonium-like Hadrons (Olsen, 1411.7738)

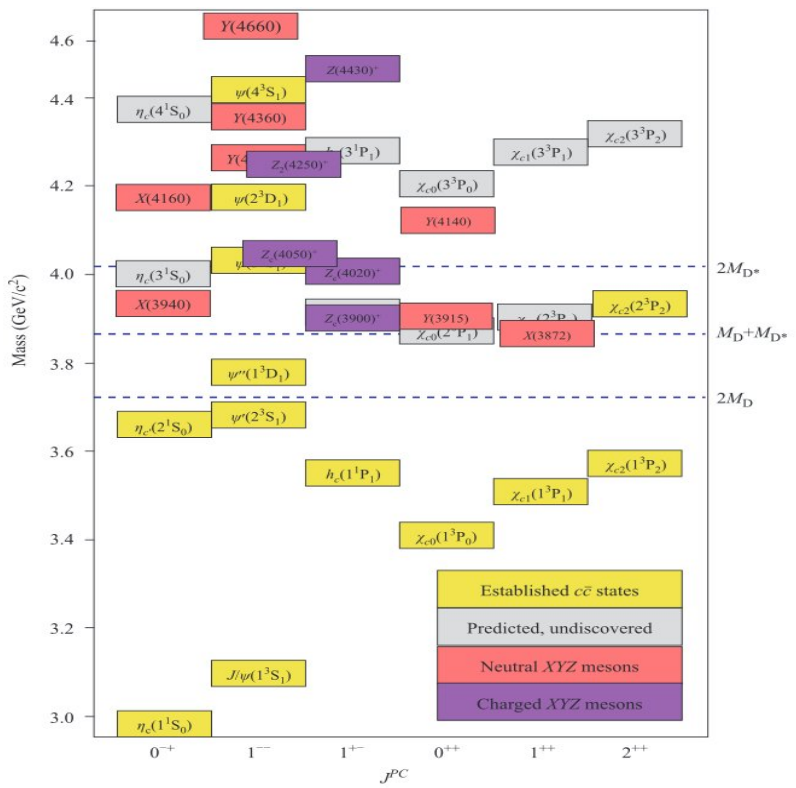




\section{Summary of Bottomonia and Bottomonium-like Hadrons (Olsen, 1411.7738)}

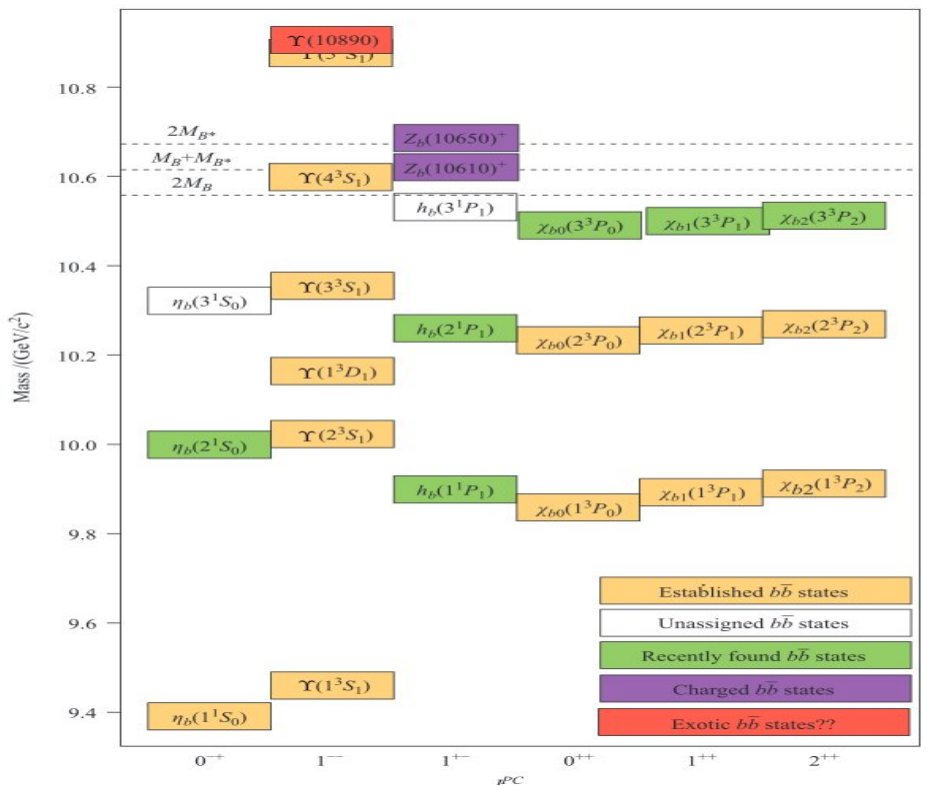


Enigmatic $Y(5 S)$ Decavs!

PRL 100, 112001 (2008) $21.7 \mathrm{fb}^{-1}$ at $10.580 \mathrm{GeV}$

$\begin{array}{lc}\Upsilon(5 S) \rightarrow \Upsilon(1 S) \pi^{+} \pi^{-} & 0.59 \pm 0.04 \pm 0.09 \\ \Upsilon(5 S) \rightarrow \Upsilon(2 S) \pi^{+} \pi^{-} & 0.85 \pm 0.07 \pm 0.16 \\ \Upsilon(5 S) \rightarrow \Upsilon(3 S) \pi^{+} \pi^{-} & 0.52_{-0.17}^{+0.20} \pm 0.10 \\ \Upsilon(2 S) \rightarrow \Upsilon(1 S) \pi^{+} \pi^{-} & 0.0060 \\ \Upsilon(3 S) \rightarrow \Upsilon(1 S) \pi^{+} \pi^{-} & 0.0009 \\ \Upsilon(4 S) \rightarrow \Upsilon(1 S) \pi^{+} \pi^{-} & 0.0019\end{array}$

PRL 102, 012001 (2009)

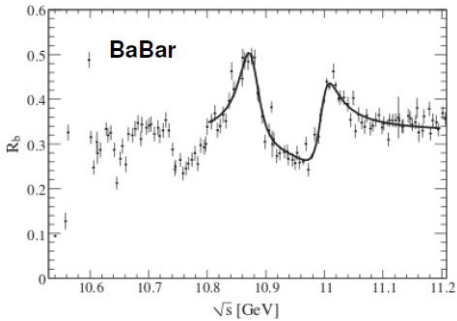

PRD 89, 091106 (2010) 1 fb - $^{-1}$ point SCAN
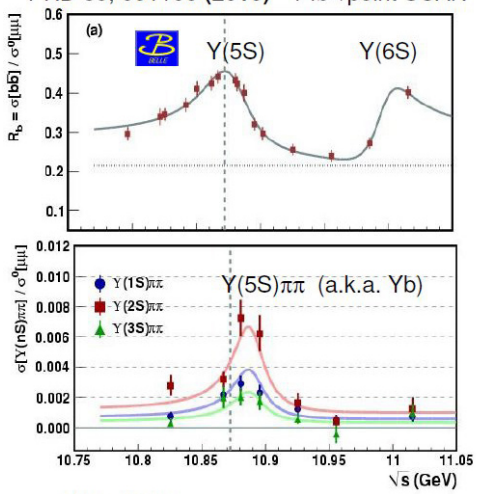

Belle 2010

$\mathrm{M}(5 \mathrm{~S}) \mathrm{b} \overline{\mathrm{b}}=10869 \pm 2 \mathrm{MeV}$

$M(5 S) \pi \pi=10888.4 \pm 2.7 \pm 1.2 \mathrm{MeV}$

$\mathrm{M}(5 \mathrm{~S})-\mathrm{M}(5 \mathrm{~S}) \pi \pi=-9 \pm 4 \mathrm{MeV}$

- Is there a $Y_{b}(10890)$ close to $\mathrm{Y}(5 S)$ ? If yes, what is it??

[AA, Hambrock, Ishtiaq Ahmed, Jamil Aslam, PLB 684 (2010) 28] 
$\sigma\left(e^{+} e^{-} \rightarrow b \bar{b}\right)$ in the $Y(10860)$ and $Y(11020)$ resonance region [Belle]

\section{$R_{b}^{\prime}$ data and fit}

- $F_{b \bar{b}}=\left|A_{n r}\right|^{2}+\left|A_{r}+A_{5 s} e^{i \phi_{5 S}} f_{5 S}+A_{6 S} e^{i \phi_{65}} f_{6 S}\right|^{2}$

- $f_{n S}=M_{n s} \Gamma_{n S} /\left[\left(s-M_{n s}^{2}\right)+i M_{n S} \Gamma_{n S}\right]$ [BW]; $A_{r}$ and $A_{n r}$ [Continuum]

- No peaking structure seen at $10.9 \mathrm{GeV}$, hinted by the BaBar data; $\Gamma\left(e^{+} e^{-}\right)<9 \mathrm{eV}(@ 90 \%$ C.L. $)$

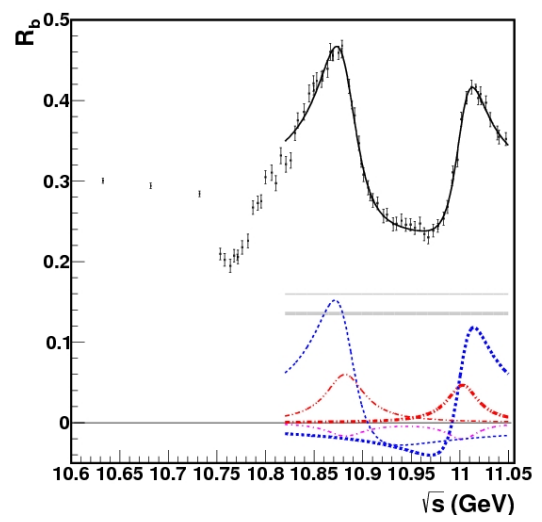


$\sigma\left(e^{+} e^{-} \rightarrow \mathrm{Y}(n S) \pi^{+} \pi^{-}\right)$in the $\mathrm{Y}(10860)$ and $\mathrm{Y}(11020)$ resonance region

[D. Santel et al. (Belle), arxiv:1501.01137 (2015)]

- Fit Values $(\mathrm{MeV}): M_{10860}=10891.1 \pm 3.2_{-1.5}^{+0.6} ; \Gamma_{10860}=53.7_{-5.6}^{+7.1}+0.9$

- $M_{5 S}(\mathrm{Y}(n S) \pi \pi)-M_{5 S}(b \bar{b})=9.2 \pm 3.4 \pm 1.9 \mathrm{MeV} ?$

Fit Values $(\mathrm{MeV}): M_{11020}=10987.5_{-2.5}^{+6.4}{ }_{-2.1}^{+9.0} ; \Gamma_{11020}=61_{-19}^{+9}+2$

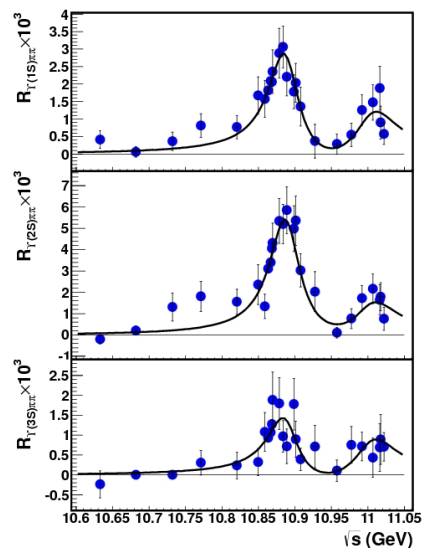


$\sigma\left(e^{+} e^{-} \rightarrow h_{b}(1 P, 2 P) \pi^{+} \pi^{-}\right)$in the $\mathrm{Y}(10860)$ and $\mathrm{Y}(11020)$ resonance region [A. Abdesselam et al. (Belle), arxiv:1508.06562 (2015)]

- Fit Values $(\mathrm{MeV}): M_{10860}=10884.7_{-2.9}^{+3.2}{ }_{-0.6}^{+8.6} ; \Gamma_{10860}=44.2_{-7.8}^{+1.9}+2.2$

- Fit Values $(\mathrm{MeV}): M_{11020}=10998.6 \pm 6.1_{-1.1}^{+16.1} ; \Gamma_{11020}=29_{-11}^{+20+2}$

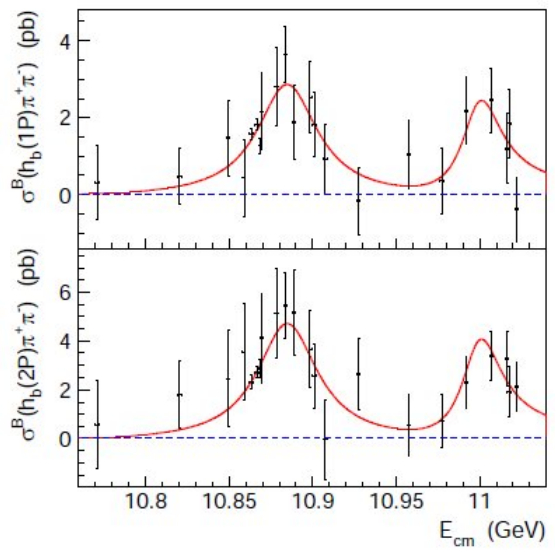


Evidence for $Z_{b}(10610)^{ \pm}$and $Z_{b}(10650)^{ \pm}($Belle $)$

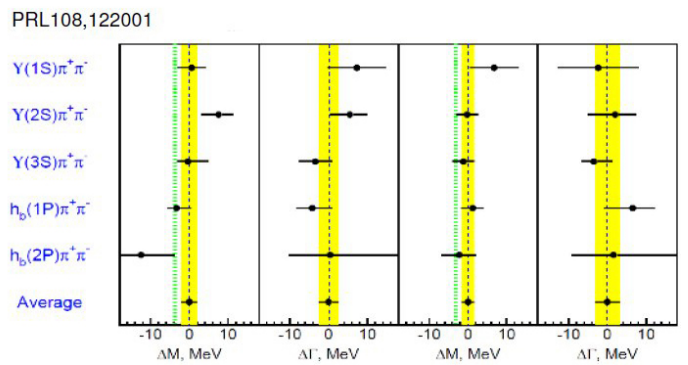

Mass and $\Gamma$ measured in 5 different final states agree

Angular analysis suggests $\mathrm{J}^{\mathrm{P}}=1^{+}$

\begin{tabular}{|l|}
\hline$Z_{b}(10610)$ \\
$M=10608$ pm $2.0 \mathrm{MeV}$ \\
$\Gamma=15.6$ pm $2.5 \mathrm{MeV}$ \\
$Z_{b}(10650)$ \\
$M=10653$ pm $1.5 \mathrm{MeV}$ \\
$\Gamma=14.4$ pm $3.2 \mathrm{MeV}$
\end{tabular}

The Di Pion transitions from the $\mathrm{Y}(5 \mathrm{~S})$ proceed via the intermediate charged state $Z_{b}$

The transition does not imply spin flip

Masses are close to $B^{*} B$ and $B^{*} B^{*}$ theresholds Molecules?

The $Y(5 S)$ is an unexpected source of $h_{b}$ 
Dipion mass distributions in $\mathrm{Y}(5 S) \rightarrow \mathrm{Y}(n S) \pi \pi$ decays?
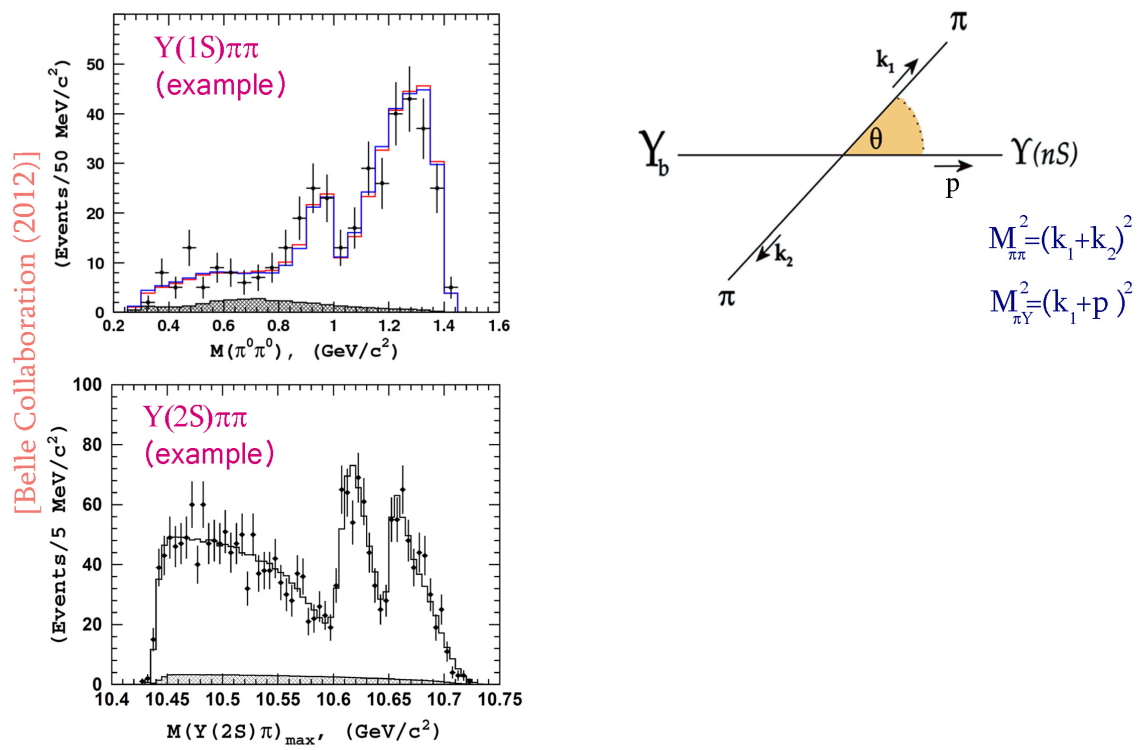
Dipion mass distributions in $\mathrm{Y}(5 S) \rightarrow \mathrm{Y}(n S) \pi \pi$ decays?
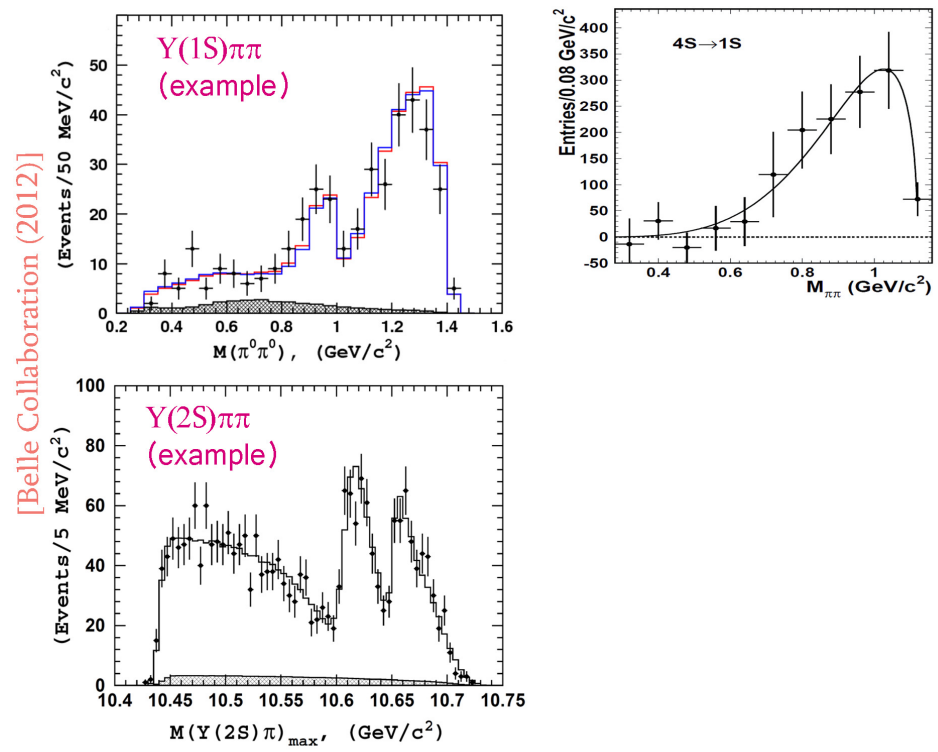
Dipion mass distributions in $\mathrm{Y}(5 S) \rightarrow \mathrm{Y}(n S) \pi \pi$ decays?
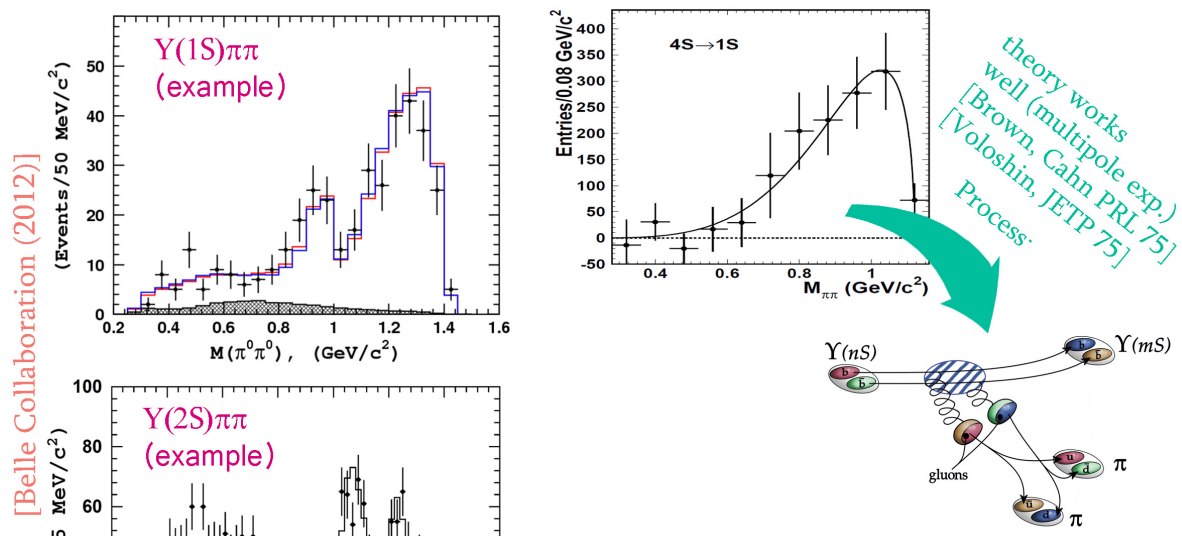
Dipion mass distributions in $\mathrm{Y}(5 S) \rightarrow \mathrm{Y}(n S) \pi \pi$ decays?
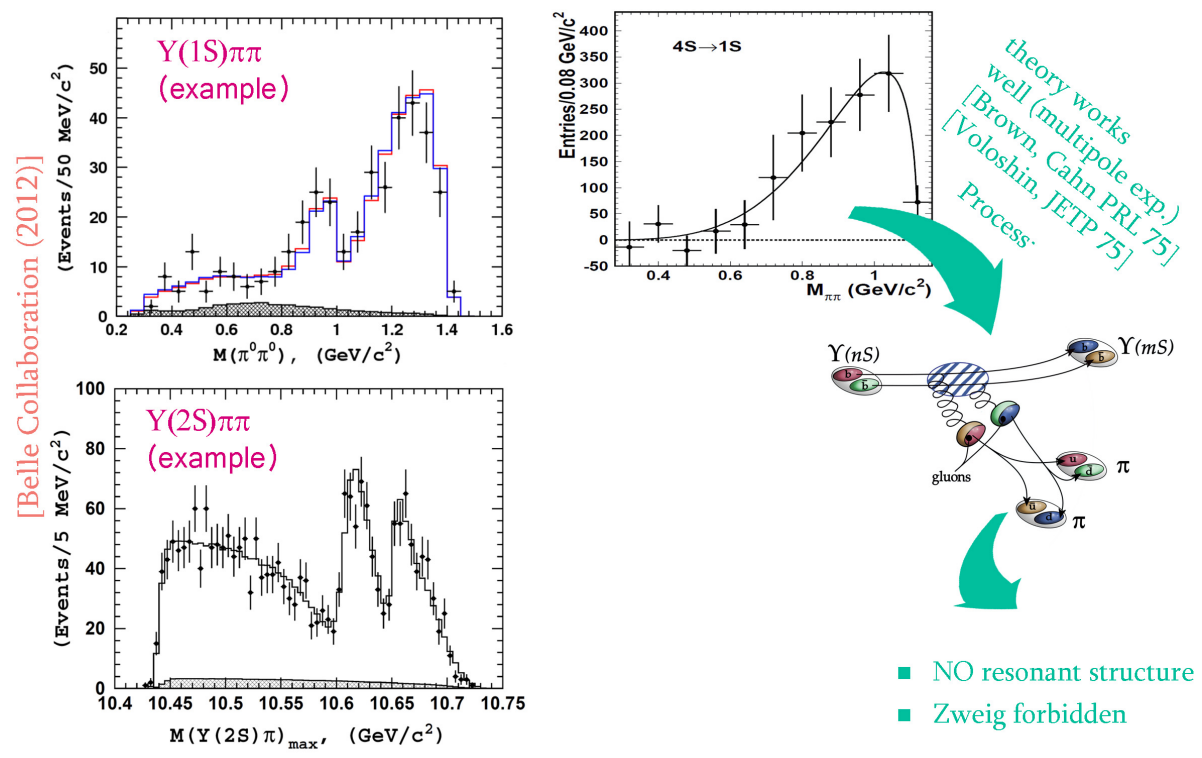
Dipion mass distributions in $\mathrm{Y}(5 S) \rightarrow \mathrm{Y}(n S) \pi \pi$ decays?
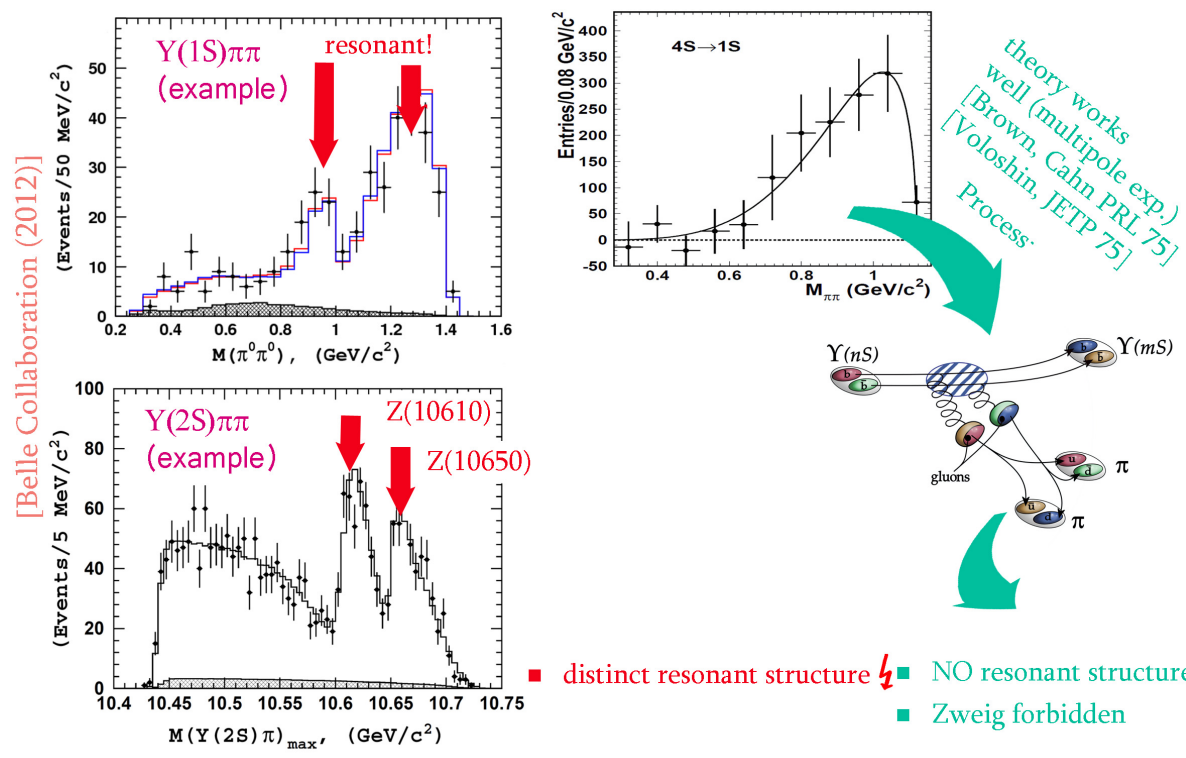

- distinct resonant structure $\{$ "NO resonant structure - Zweig forbidden 
Dipion mass distributions in $\mathrm{Y}(5 S) \rightarrow \mathrm{Y}(n S) \pi \pi$ decays?
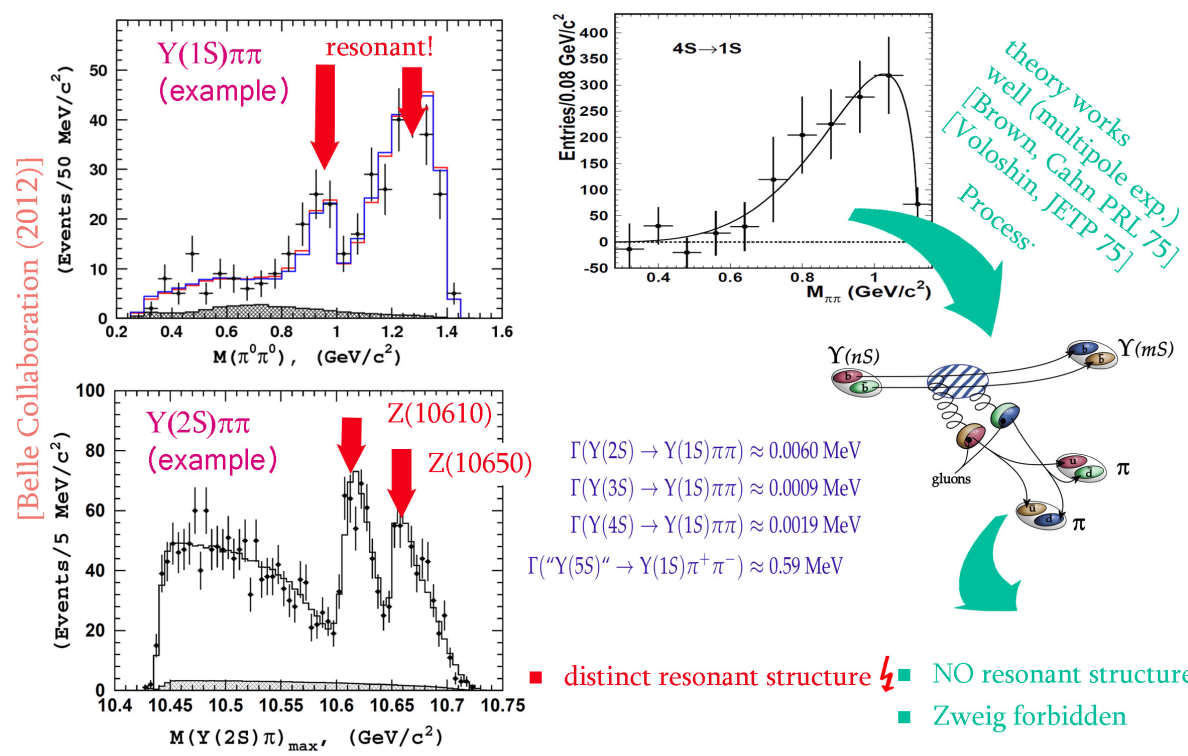

$$
\begin{aligned}
\Gamma(\mathrm{Y}(2 S) \rightarrow \mathrm{Y}(1 S) \pi \pi) & \approx 0.0060 \mathrm{MeV} \\
\Gamma(\mathrm{Y}(3 S) \rightarrow \mathrm{Y}(1 S) \pi \pi) & \approx 0.0009 \mathrm{MeV} \\
\Gamma(\mathrm{Y}(4 S) \rightarrow \mathrm{Y}(1 S) \pi \pi) & \approx 0.0019 \mathrm{MeV} \\
\Gamma\left({ }^{\prime} \mathrm{Y}(5 S)^{\prime \prime} \rightarrow \mathrm{Y}(1 S) \pi^{+} \pi^{-}\right) & \approx 0.59 \mathrm{MeV}
\end{aligned}
$$$$
\Upsilon(n S)
$$$$
Y(m S)
$$

- distinct resonant structure $\boldsymbol{q}$ - NO resonant structure

- Zweig forbidden 
Dipion mass distributions in $\mathrm{Y}(5 S) \rightarrow \mathrm{Y}(n S) \pi \pi$ decays?

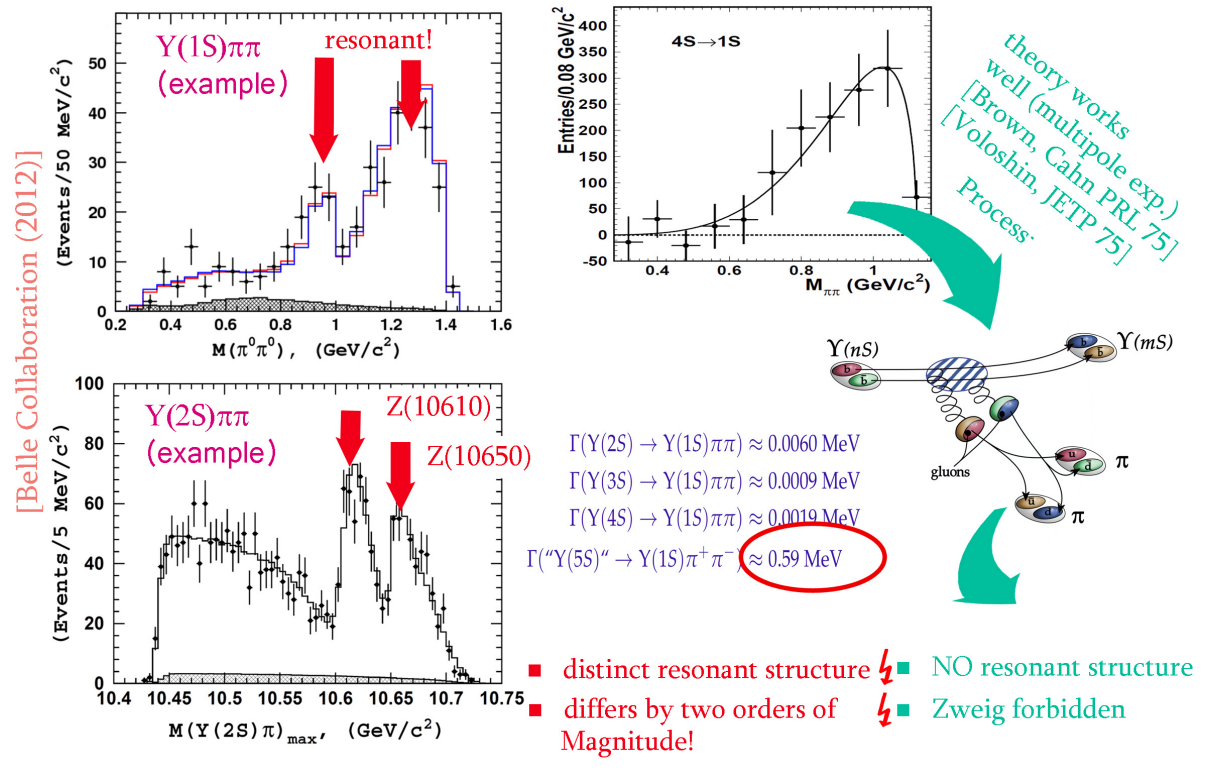


Dipion mass distributions in $\mathrm{Y}(5 S) \rightarrow \mathrm{Y}(n S) \pi \pi$ decays?

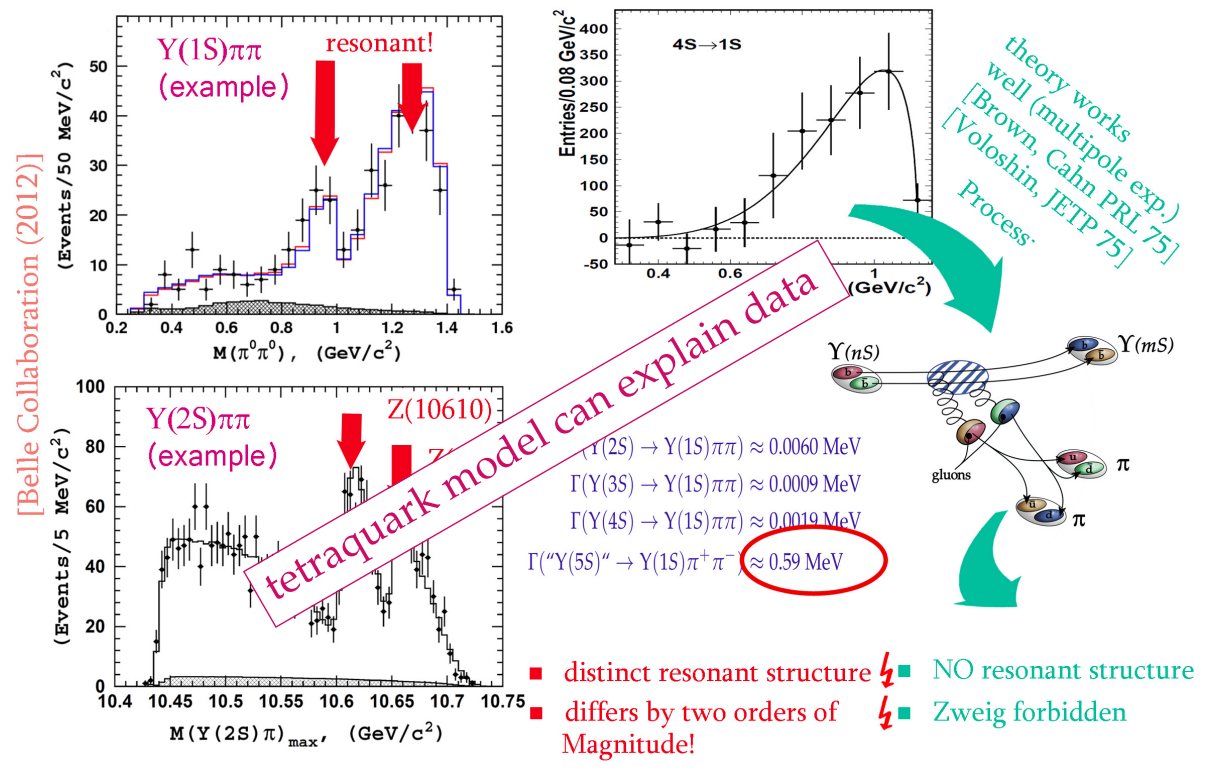


Models for XYZ Mesons

Quarkonium Tetraquarks

- compact tetraquark $\begin{array}{ll}Q & \bar{q} \\ q & \bar{Q}\end{array}$

- meson molecule

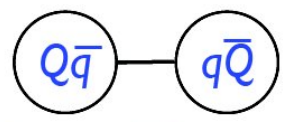

- diquark-onium

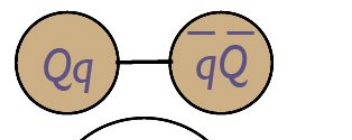

- hadro-quarkonium ( $\left.{ }^{q} \mathrm{QQ}\right)$

- quarkonium adjoint meson

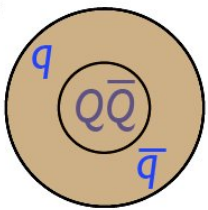




\section{$X, Y, Z$ Exotics}

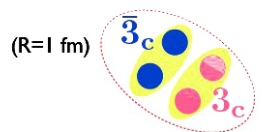

a compact 'tetraquark'

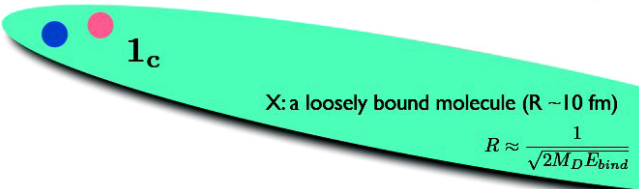

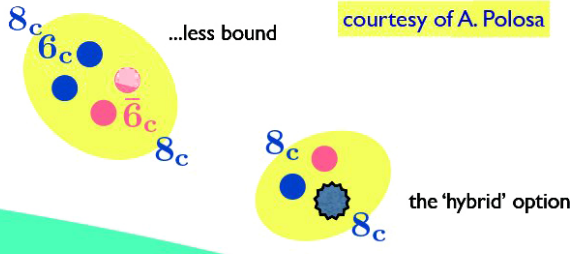

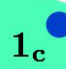

\section{Hadro-charmonium}

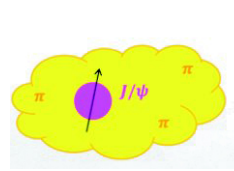

Aoloshin arXiv:1304.0380
A $c \bar{c}$ state surrounded by light matter
Decay into $\eta_{c} \rho$ forbidden by HQSS




\section{Diquarks: Color Representation}

- One gluon exchange model [Jaffe,Phys.Rept.(2005)]

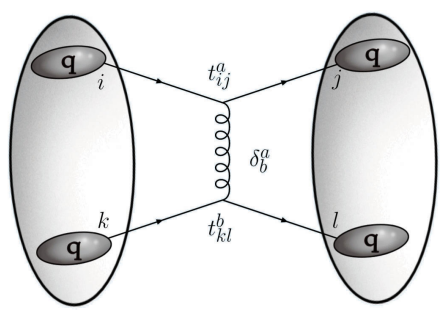




\section{Diquarks: Color Representation}

- One gluon exchange model [Jaffe,Phys.Rept.(2005)]

Color factor determines binding: Negative sign $\rightarrow$ Attractive

- Quarks in diquark transform as:

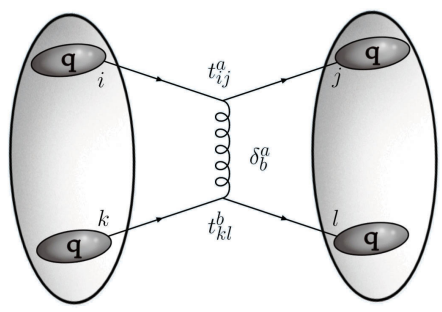

\section{$3 \otimes 3=\overline{3} \oplus 6$}




\section{Diquarks: Color Representation}

One gluon exchange model [Jaffe,Phys.Rept.(2005)]

Color factor determines binding:

Negative sign $\rightarrow$ Attractive

Quarks in diquark transform as:

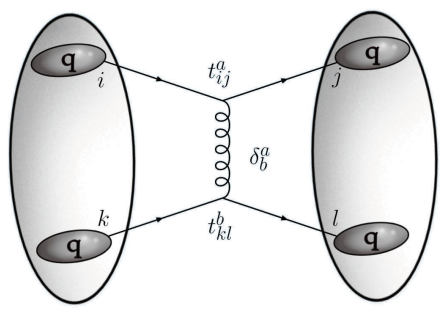

\section{$3 \otimes 3=\overline{3} \oplus 6$}

qq bound state color factor:

$$
t_{i j}^{a} t_{k l}^{a}=-\frac{2}{3} \underbrace{\left(\delta_{i j} \delta_{k l}-\delta_{i l} \delta_{k j}\right) / 2}_{\text {antisymmetric: projects }}+\frac{1}{3} \underbrace{\left(\delta_{i j} \delta_{k l}+\delta_{i l} \delta_{k j}\right) / 2}_{\text {symmetric: projects } 6}
$$




\section{Diquarks: Color Representation}

One gluon exchange model [Jaffe,Phys.Rept.(2005)]

Color factor determines binding:

Negative sign $\rightarrow$ Attractive

Quarks in diquark transform as:

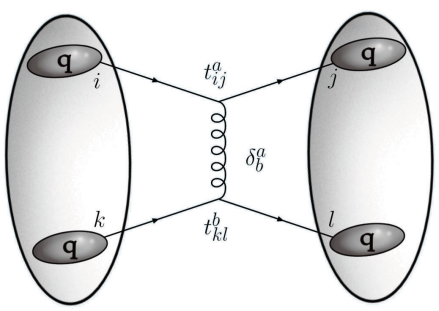

\section{$3 \otimes 3=\overline{3} \oplus 6$}

qq bound state color factor:

$$
t_{i j}^{a} t_{k l}^{a}=(-\frac{2}{3} \underbrace{\left(\delta_{i j} \delta_{k l}-\delta_{i l} \delta_{k j}\right) / 2}_{\text {antisymmetric: projects }}+\frac{1}{3} \underbrace{\left(\delta_{i j} \delta_{k l}+\delta_{i l} \delta_{k j}\right) / 2}_{\text {symmetric: projects } 6}
$$




\section{Diquarks: Color Representation}

- One gluon exchange model [Jaffe,Phys.Rept.(2005)]

Color factor determines binding:

Negative sign $\rightarrow$ Attractive

Quarks in diquark transform as:

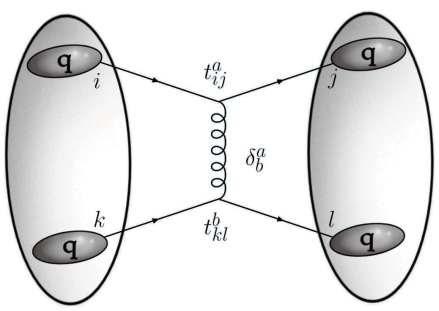

$$
3 \otimes 3=\overline{3} \oplus \not<
$$

- $q q$ bound state color factor:

$$
t_{i j}^{a} t_{k l}^{a}=(-\frac{2}{3} \underbrace{\left(\delta_{i j} \delta_{k l}-\delta_{i l} \delta_{k j}\right) / 2}_{\text {antisymmetric: projects }} \underbrace{+\frac{1}{3} \underbrace{\left(\delta_{i j} \delta_{k l}+\delta_{i l} \delta_{k j}\right) / 2}}_{\text {symmetric: projects } 6}
$$


Diquarks: Spin representation
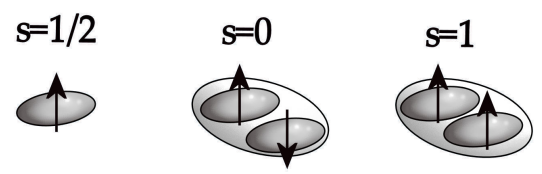


\section{Diquarks: Spin representation}
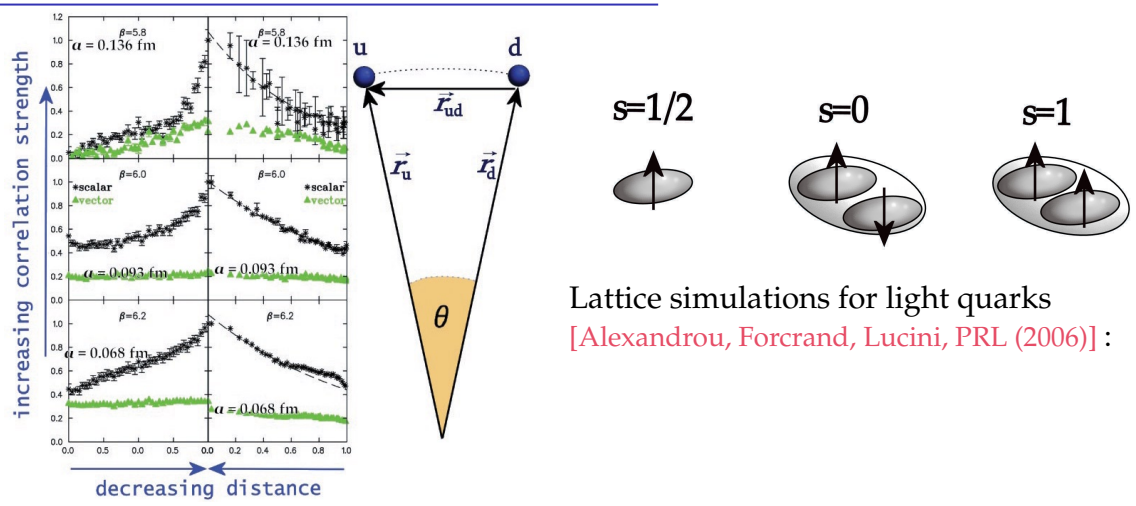

Lattice simulations for light quarks [Alexandrou, Forcrand, Lucini, PRL (2006)] :

- Calculation of 2 quark correlation strength

Decreasing distance

$\rightarrow$ Increasing strength for "good" diquarks

Diquark size $\mathcal{O}(1 \mathrm{fm})$ 


\section{Diquarks: Spin representation}
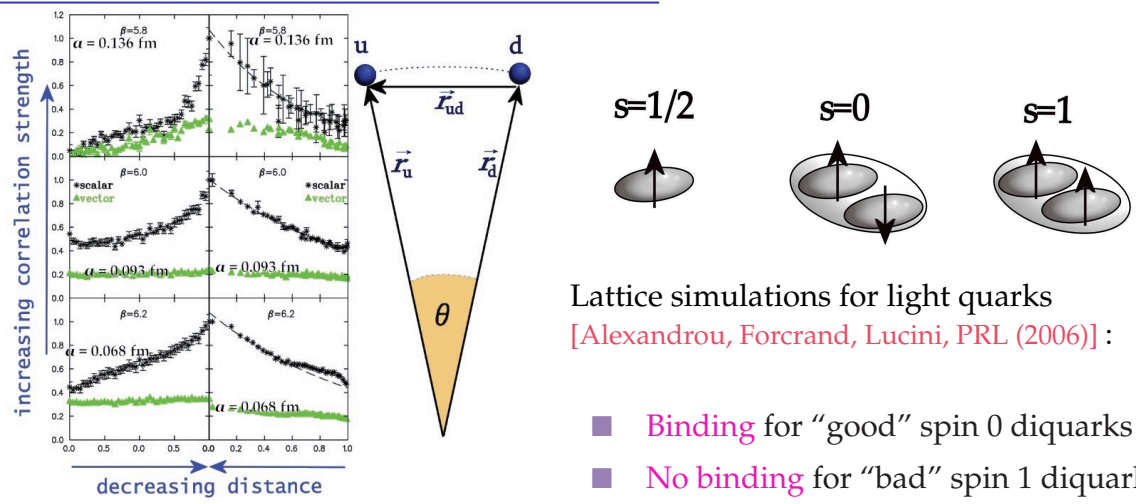

Lattice simulations for light quarks [Alexandrou, Forcrand, Lucini, PRL (2006)] :

- Binding for "good" spin 0 diquarks

- No binding for "bad" spin 1 diquarks

- Calculation of 2 quark correlation strength

Spin decoupling in HQ-Limit

- Decreasing distance

$\rightarrow$ Increasing strength for "good" diquarks

"Bad" diquarks in $b$-sector might bind

niquark size $\mathcal{O}(1 \mathrm{fm})$ 
Diquark Model of Tetra- and Pentaquarks

Diquarks and Anti-diquarks are the building blocks of Tetraquarks Color representation: $3 \otimes 3=\overline{3} \oplus 6$; only $\overline{3}$ is attractive; $C_{\overline{3}}=1 / 2 C_{3}$ Interpolating diquark operators for the two spin-states of diquarks Scalar: $0^{+} \mathcal{Q}_{i \alpha}=\epsilon_{\alpha \beta \gamma}\left(\bar{b}_{c}^{\beta} \gamma_{5} q_{i}^{\gamma}-\bar{q}_{i_{c}}^{\beta} \gamma_{5} b^{\gamma}\right)$ Axial-Vector: $\quad 1^{+} \quad \overrightarrow{\mathcal{Q}}_{i \alpha}=\epsilon_{\alpha \beta \gamma}\left(\bar{b}_{c}^{\beta} \vec{\gamma} q_{i}^{\gamma}+\bar{q}_{i_{c}}^{\beta} \vec{\gamma} b^{\gamma}\right)$ 
Diquark Model of Tetra- and Pentaquarks

Diquarks and Anti-diquarks are the building blocks of Tetraquarks Color representation: $3 \otimes 3=\overline{3} \oplus 6$; only $\overline{3}$ is attractive; $C_{\overline{3}}=1 / 2 C_{3}$

Interpolating diquark operators for the two spin-states of diquarks

$$
\text { Scalar: } 0^{+} \quad \mathcal{Q}_{i \alpha}=\epsilon_{\alpha \beta \gamma}\left(\bar{b}_{c}^{\beta} \gamma_{5} q_{i}^{\gamma}-\bar{q}_{i_{c}}^{\beta} \gamma_{5} b^{\gamma}\right)
$$

Axial-Vector: $1^{+} \quad \overrightarrow{\mathcal{Q}}_{i \alpha}=\epsilon_{\alpha \beta \gamma}\left(\bar{b}_{c}^{\beta} \vec{\gamma} q_{i}^{\gamma}+\bar{q}_{i_{c}}^{\beta} \vec{\gamma}^{\gamma}\right)$

NR limit: States parametrized by Pauli matrices :

$$
\text { Scalar: } 0^{+} \Gamma^{0}=\frac{\sigma_{2}}{\sqrt{2}}
$$

Axial-Vector: $1^{+} \quad \vec{\Gamma}=\frac{\sigma_{2} \vec{\sigma}}{\sqrt{2}}$ 
Diquark Model of Tetra- and Pentaquarks

Diquarks and Anti-diquarks are the building blocks of Tetraquarks Color representation: $3 \otimes 3=\overline{3} \oplus 6$; only $\overline{3}$ is attractive; $C_{\overline{3}}=1 / 2 C_{3}$

Interpolating diquark operators for the two spin-states of diquarks

$$
\text { Scalar: } 0^{+} \quad \mathcal{Q}_{i \alpha}=\epsilon_{\alpha \beta \gamma}\left(\bar{b}_{c}^{\beta} \gamma_{5} q_{i}^{\gamma}-\bar{q}_{i_{c}}^{\beta} \gamma_{5} b^{\gamma}\right)
$$

Axial-Vector: $1^{+} \quad \overrightarrow{\mathcal{Q}}_{i \alpha}=\epsilon_{\alpha \beta \gamma}\left(\bar{b}_{c}^{\beta} \vec{\gamma} q_{i}^{\gamma}+\bar{q}_{i_{c}}^{\beta} \vec{\gamma}^{\gamma}\right)$

NR limit: States parametrized by Pauli matrices:

$$
\text { Scalar: } 0^{+} \Gamma^{0}=\frac{\sigma_{2}}{\sqrt{2}}
$$

$$
\text { Axial-Vector: } 1^{+} \quad \vec{\Gamma}=\frac{\sigma_{2} \vec{\sigma}}{\sqrt{2}}
$$

Diquark spin $s_{\mathcal{Q}} \rightarrow$ tetraquark total angular momentum J:

$$
\left|Y_{[b q]}\right\rangle=\left|s_{\mathcal{Q}}, s_{\overline{\mathcal{Q}}} ; J\right\rangle
$$

Tetraquarks:

$$
\begin{aligned}
\left|0_{\mathcal{Q}}, 0_{\overline{\mathcal{Q}}} ; 0_{J}\right\rangle & =\Gamma^{0} \otimes \Gamma^{0} \\
\left|1_{\mathcal{Q}}, 1_{\overline{\mathcal{Q}}} ; 0_{J}\right\rangle & =\frac{1}{\sqrt{3}} \Gamma^{i} \otimes \Gamma_{i} \ldots \\
\left|0_{\mathcal{Q}}, 1_{\overline{\mathcal{Q}}} ; 1_{J}\right\rangle & =\Gamma^{0} \otimes \Gamma^{i}
\end{aligned}
$$


NR Hamiltonian for Tetraquarks with hidden charm

States need to diagonalize Hamiltonian:

$$
H=2 m_{\mathcal{Q}}+H_{S S}^{(q q)}+H_{S S}^{(q \bar{q})}+H_{S L}+H_{L L}
$$

$$
\begin{aligned}
H_{\mathrm{eff}}(X, Y, Z) & =2 m_{\mathcal{Q}}+\frac{B_{Q}}{2}\left\langle L^{2}\right\rangle-2 a\langle L \cdot S\rangle+2 \kappa_{q Q}\left[\left\langle s_{q} \cdot s_{Q}\right\rangle+\left\langle s_{\bar{q}} \cdot s_{\bar{Q}}\right\rangle\right] \\
& =2 m_{\mathcal{Q}}-a J(J+1)+\left(\frac{B_{Q}}{2}+a\right) L(L+1)+a S(S+1)-3 \kappa_{q Q} \\
& +\kappa_{q Q}\left[s_{q Q}\left(s_{q Q}+1\right)+s_{\bar{q} \bar{Q}}\left(s_{\bar{q} \bar{Q}}+1\right)\right]
\end{aligned}
$$


NR Hamiltonian for Tetraquarks with hidden charm States need to diagonalize Hamiltonian:

$$
H=2 m_{\mathcal{Q}}+H_{S S}^{(q q)}+H_{S S}^{(q \bar{q})}+H_{S L}+H_{L L}
$$

with

\section{constituent mass}

$$
\begin{aligned}
H_{\mathrm{eff}}(X, Y, Z) & =2 m_{\mathcal{Q}}+\frac{B_{Q}}{2}\left\langle L^{2}\right\rangle-2 a\langle L \cdot S\rangle+2 \kappa_{q Q}\left[\left\langle s_{q} \cdot s_{Q}\right\rangle+\left\langle s_{\bar{q}} \cdot s_{\bar{Q}}\right\rangle\right] \\
& =2 m_{\mathcal{Q}}-a J(J+1)+\left(\frac{B_{Q}}{2}+a\right) L(L+1)+a S(S+1)-3 \kappa_{q Q} \\
& +\kappa_{q Q}\left[s_{q Q}\left(s_{q Q}+1\right)+s_{\bar{q} \bar{Q}}\left(s_{\bar{q} \bar{Q}}+1\right)\right]
\end{aligned}
$$


NR Hamiltonian for Tetraquarks with hidden charm States need to diagonalize Hamiltonian:

$$
H=2 m_{\mathcal{Q}}+H_{S S}^{(q q)}+H_{S S}^{(q \bar{q} \boldsymbol{k}}+H_{S L}+H_{L L}
$$

with

$$
\text { qq spin coupling }
$$

\section{$q \bar{q}$ spin coupling}

$$
\begin{aligned}
H_{S S}^{(q q)}= & 2\left(\mathcal{K}_{c q}\right) \overline{\overline{3}}\left[\left(\mathbf{S}_{c} \cdot \mathbf{S}_{q}\right)+\left(\mathbf{S}_{\bar{c}} \cdot \mathbf{S}_{\bar{q}}\right)\right] \\
H_{S S}^{(q \bar{q})}= & 2\left(\mathcal{K}_{c \bar{q}}\right)\left(\mathbf{S}_{c} \cdot \mathbf{S}_{\bar{q}}+\mathbf{S}_{\bar{c}} \cdot \mathbf{S}_{q}\right) \\
& +2 \mathcal{K}_{c \bar{c}}\left(\mathbf{S}_{c} \cdot \mathbf{S}_{\bar{c}}\right)+2 \mathcal{K}_{q \bar{q}}\left(\mathbf{S}_{q} \cdot \mathbf{S}_{\bar{q}}\right)
\end{aligned}
$$

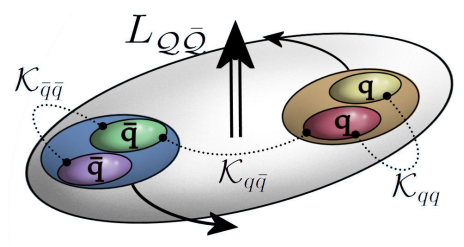

$$
\begin{aligned}
H_{\mathrm{eff}}(X, Y, Z) & =2 m_{\mathcal{Q}}+\frac{B_{Q}}{2}\left\langle L^{2}\right\rangle-2 a\langle L \cdot S\rangle+2 \kappa_{q Q}\left[\left\langle s_{q} \cdot s_{Q}\right\rangle+\left\langle s_{\bar{q}} \cdot s_{\bar{Q}}\right\rangle\right] \\
& =2 m_{\mathcal{Q}}-a J(J+1)+\left(\frac{B_{Q}}{2}+a\right) L(L+1)+a S(S+1)-3 \kappa_{q Q} \\
& +\kappa_{q Q}\left[s_{q Q}\left(s_{q Q}+1\right)+s_{\bar{q} \bar{Q}}\left(s_{\bar{q} \bar{Q}}+1\right)\right]
\end{aligned}
$$


NR Hamiltonian for Tetraquarks with hidden charm States need to diagonalize Hamiltonian:

with

$$
\begin{aligned}
& H=2 m_{\mathcal{Q}}+H_{S S}^{(q q)}+H_{S S}^{(q \bar{q})}+H_{g} \nearrow 4+H_{L L} \\
& \text { LS coupling LL coupling }
\end{aligned}
$$

$$
\begin{aligned}
H_{S S}^{(q q)}= & 2\left(\mathcal{K}_{c q}\right)_{\overline{3}}\left[\left(\mathbf{S}_{c} \cdot \mathbf{S}_{q}\right)+\left(\mathbf{S}_{\bar{c}} \cdot \mathbf{S}_{\bar{q}}\right)\right] \\
H_{S S}^{(q \bar{q})}= & 2\left(\mathcal{K}_{c \bar{q}}\right)\left(\mathbf{S}_{c} \cdot \mathbf{S}_{\bar{q}}+\mathbf{S}_{\bar{c}} \cdot \mathbf{S}_{q}\right) \\
& +2 \mathcal{K}_{c \bar{c}}\left(\mathbf{S}_{c} \cdot \mathbf{S}_{\bar{c}}\right)+2 \mathcal{K}_{q \bar{q}}\left(\mathbf{S}_{q} \cdot \mathbf{S}_{\bar{q}}\right) \\
H_{S L}= & 2 A_{\mathcal{Q}}\left(\mathbf{S}_{\mathcal{Q}} \cdot \mathbf{L}+\mathbf{S}_{\overline{\mathcal{Q}}} \cdot \mathbf{L}\right) \\
H_{L L}= & B_{\mathcal{Q}} \frac{L_{\mathcal{Q} \overline{\mathcal{Q}}}\left(L_{\mathcal{Q} \overline{\mathcal{Q}}}+1\right)}{2} \\
H_{\text {eff }}(X, Y, Z)= & 2 m_{\mathcal{Q}}+\frac{B_{Q}}{2}\left\langle L^{2}\right\rangle-2 a\langle L \cdot S\rangle+2 \kappa_{q Q}\left[\left\langle s_{q} \cdot s_{Q}\right\rangle+\left\langle s_{\bar{q}} \cdot s_{\bar{Q}}\right\rangle\right] \\
= & 2 m_{\mathcal{Q}}-a J(J+1)+\left(\frac{B_{Q}}{2}+a\right) L(L+1)+a S(S+1)-3 \kappa_{q Q} \\
& +\kappa_{q Q}\left[s_{q Q}\left(s_{q Q}+1\right)+s_{\bar{q} \bar{Q}}\left(s_{\bar{q} \bar{Q}}+1\right)\right]
\end{aligned}
$$

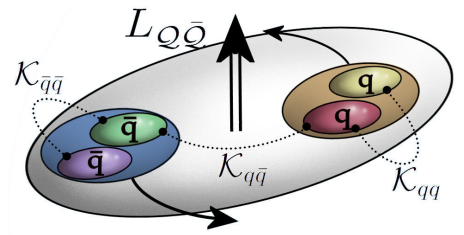


Low-lying $S$ - and $P$-wave Tetraquark States

\section{$S$-wave states}

- In the $\left|s_{q Q}, s_{\bar{q} \bar{Q}} ; S, L\right\rangle_{J}$ and $\left|s_{q \bar{q}}, s_{Q \bar{Q}} ; S^{\prime}, L^{\prime}\right\rangle_{J}$ bases, the positive parity $S$-wave tetraquarks are given in terms of the six states listed below (charge conjugation is defined for neutral states)

\begin{tabular}{clccc}
\hline \hline Label & $J^{P C}$ & $\left|s_{q Q}, s_{\bar{q} \bar{Q}} ; S, L\right\rangle_{J}$ & $\left|s_{q \bar{q}}, s_{Q \bar{Q}^{\circ}} ; S^{\prime}, L^{\prime}\right\rangle_{J}$ & Mass \\
\hline$X_{0}$ & $0^{++}$ & $|0,0 ; 0,0\rangle_{0}$ & $\left(|0,0 ; 0,0\rangle_{0}+\sqrt{3}|1,1 ; 0,0\rangle_{0}\right) / 2$ & $M_{00}-3 \kappa_{q Q}$ \\
$X_{0}^{\prime}$ & $0^{++}$ & $|1,1 ; 0,0\rangle_{0}$ & $\left(\sqrt{3}|0,0 ; 0,0\rangle_{0}-|1,1 ; 0,0\rangle_{0}\right) / 2$ & $M_{00}+\kappa_{q Q}$ \\
$X_{1}$ & $1^{++}$ & $\left(|1,0 ; 1,0\rangle_{1}+|0,1 ; 1,0\rangle_{1}\right) / \sqrt{2}$ & $\left|1,1 ; 1, L^{\prime}\right\rangle_{1}$ & $M_{00}-\kappa_{q Q}$ \\
$Z$ & $1^{+-}$ & $\left.|1,0 ; 1,0\rangle_{1}-|0,1 ; 1,0\rangle_{1}\right) / \sqrt{2}$ & $\left(\left|1,0 ; 1, L^{\prime}\right\rangle_{1}-\left|0,1 ; 1, L^{\prime}\right\rangle_{1}\right) / \sqrt{2}$ & $M_{00}-\kappa_{q Q}$ \\
$Z^{\prime}$ & $1^{+-}$ & $|1,1 ; 1,0\rangle_{1}$ & $\left(\left|1,0 ; 1, L^{\prime}\right\rangle_{1}+\left|0,1 ; 1, L^{\prime}\right\rangle_{1}\right) / \sqrt{2}$ & $M_{00}+\kappa_{q Q}$ \\
$X_{2}$ & $2^{++}$ & $|1,1 ; 2,0\rangle_{2}$ & $\left|1,1 ; 2, L^{\prime}\right\rangle_{2}$ & $M_{00}+\kappa_{q Q}$ \\
\hline \hline
\end{tabular}

$P$-wave $\left(J^{P C}=1^{--}\right)$states

\begin{tabular}{ccccc}
\hline \hline Label & $J^{P C}$ & $\left|s_{q Q}, s_{\bar{q} \bar{Q}} ; S, L\right\rangle_{J}$ & $\left|s_{q \bar{q}}, s_{Q \bar{Q}} ; S^{\prime}, L^{\prime}\right\rangle_{J}$ & Mass \\
\hline$Y_{1}$ & $1^{--}$ & $|0,0 ; 0,1\rangle_{1}$ & $\left(|0,0 ; 0,1\rangle_{1}+\sqrt{3}|1,1 ; 0,1\rangle_{1}\right) / 2$ & $M_{00}-3 \kappa_{q Q}+B_{Q}$ \\
$Y_{2}$ & $1^{--}$ & $\left(|1,0 ; 1,1\rangle_{1}+|0,1 ; 1,1\rangle_{1}\right) / \sqrt{2}$ & $\left|1,1 ; 1, L^{\prime}\right\rangle_{1}$ & $M_{00}-\kappa_{q Q}+2 a+B_{Q}$ \\
$Y_{3}$ & $1^{--}$ & $|1,1 ; 0,1\rangle_{1}$ & $\left(\sqrt{3}|0,0 ; 0,1\rangle_{1}-|1,1 ; 0,1\rangle_{1}\right) / 2$ & $M_{00}+\kappa_{q Q}+B_{Q}$ \\
$Y_{4}$ & $1^{--}$ & $|1,1 ; 2,1\rangle_{1}$ & $\left|1,1 ; 2, L^{\prime}\right\rangle_{1}$ & $M_{00}+\kappa_{q Q}+6 a+B_{Q}$ \\
$Y_{5}$ & $1^{--}$ & $|1,1 ; 2,3\rangle_{1}$ & $\left|1,1 ; 2, L^{\prime}\right\rangle_{1}$ & $M_{00}+\kappa_{q Q}+16 a+6 B_{Q}$ \\
\hline \hline
\end{tabular}


Charmonium-like and Bottomonium-like Tetraquark Spectrum

\section{Parameters in the Mass Formula \\ (with Satoshi Mishima)}

\begin{tabular}{c|cc}
\hline \hline & charmonium-like & bottomonium-like \\
\hline$M_{00}[\mathrm{MeV}]$ & 3957 & 10630 \\
$\kappa_{q Q}[\mathrm{MeV}]$ & 67 & 22.5 \\
$B_{Q}[\mathrm{MeV}]$ & 268 & 329 \\
$a[\mathrm{MeV}]$ & 52.5 & 26 \\
\hline \hline
\end{tabular}

\begin{tabular}{c|c|cc|cc}
\hline \hline & \multirow{2}{*}{ Label } & $J^{P C}$ & \multicolumn{2}{|c|}{ charmonium-like } & \multicolumn{2}{c}{ bottomonium-like } \\
\hline$X_{0}$ & $0^{++}$ & - & Mass $[\mathrm{MeV}]$ & State & Mass $[\mathrm{MeV}]$ \\
$X_{0}^{\prime}$ & $0^{++}$ & - & 3756 & - & 10562.2 \\
$X_{1}$ & $1^{++}$ & $X(3872)$ & 4024 & - & 10652 \\
$Z$ & $1^{+-}$ & $Z_{c}^{+}(3900)$ & 3890 & - & 10607 \\
$Z^{\prime}$ & $1^{+-}$ & $Z_{c}^{+}(4020)$ & 3890 & $Z_{b}^{+, 0}(10610)$ & 10607 \\
$X_{2}$ & $2^{++}$ & - & 4024 & $Z_{b}^{+}(10650)$ & 10652 \\
\hline$Y_{1}$ & $1^{--}$ & $Y(4008)$ & 4024 & - & 10652 \\
$Y_{2}$ & $1^{--}$ & $Y(4260)$ & 4024 & $Y_{b}(10891)$ & 10891 \\
$Y_{3}$ & $1^{--}$ & $Y(4290)($ or $Y(4220))$ & 4263 & $Y_{b}(10987)$ & 10987 \\
$Y_{4}$ & $1^{--}$ & $Y(4630)$ & 4292 & - & 10981 \\
$Y_{5}$ & $1^{--}$ & - & 4607 & - & 11135 \\
\hline \hline
\end{tabular}


Comparison with current data in the Charmonium-like sector

- Better agreement with data achieved with more tightly-bound quarks inside a diquark than is the case for diquarks in baryons [Maiani et al. (2014)]

- - - - New; - . . - - Old

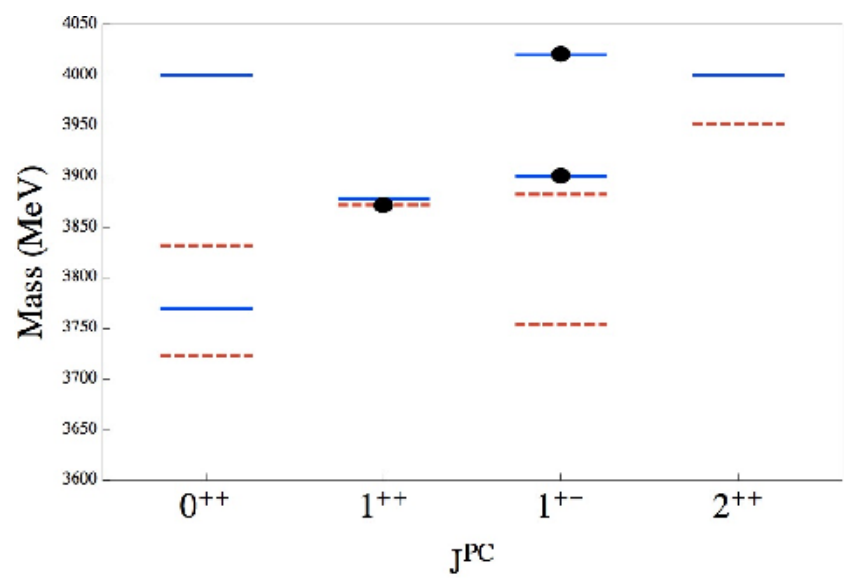




\section{$[b q][\bar{b} \bar{q}]$ Constituent Model Spectrum}

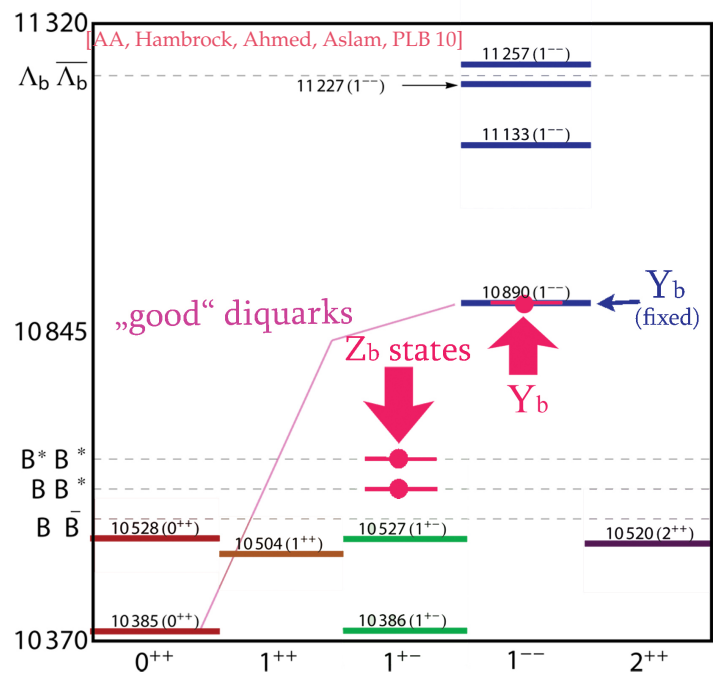

- states are iso-doublets $q=u, d$

With the old Maiani et al. Paradigm, tetraquark $Z_{b}$ masses do not agree with Belle

- However tetraquarks with mixing \& self energy corrections in principle allowed in parts of parameter space $[A A$, Hambrock, Wang, PRD 11] ,

- but with the new Maiani et al. paradigm, $M\left(Z_{b}\right)-M\left(Z_{b}^{\prime}\right)$ fixes $2 \kappa_{q b}=45 \mathrm{MeV} \sim 2 \kappa_{q c}$ ，

in agreement with the heavy quark symmetry: $\kappa_{q b} / \kappa_{q c} \simeq m_{c} / m_{b}$ 
Heavy-Quark-Spin Flip in $\mathrm{Y}(10890) \rightarrow Z_{b} / Z_{b}^{\prime}+\pi \rightarrow h_{b}(1 P, 2 P) \pi \pi$

A.A., L. Maiani, A.D. Polosa, V. Riquer; PR D91, 017502 (2015)

Relative normalizations and phases for $s_{b \bar{b}}: 1 \rightarrow 1$ and $1 \rightarrow 0$ transitions

\begin{tabular}{|c|c|c|c|c|c|}
\hline Final State & $\mathrm{Y}(1 S) \pi^{+} \pi^{-}$ & $\mathrm{Y}(2 S) \pi^{+} \pi^{-}$ & $\mathrm{Y}(3 S) \pi^{+} \pi^{-}$ & $h_{b}(1 P) \pi^{+} \pi^{-}$ & $h_{b}(2 P) \pi^{+} \pi^{-}$ \\
\hline \hline Rel. Norm. & $0.57 \pm 0.21_{-0.04}^{+0.19}$ & $0.86 \pm 0.11_{-0.10}^{+0.04}$ & $0.96 \pm 0.14_{-0.05}^{+0.08}$ & $1.39 \pm 0.37_{-0.15}^{+0.05}$ & $1.6_{-0.4-0.6}^{+0.6+0.4}$ \\
\hline Rel. Phase & $58 \pm 43_{-9}^{+4}$ & $-13 \pm 13_{-8}^{+17}$ & $-9 \pm 19_{-26}^{+11}$ & $187_{-57-12}^{+44+3}$ & $181_{-105-109}^{+65+74}$ \\
\hline
\end{tabular}

- In $Y(10890), S_{b \bar{b}}=1$. In $h_{b}(n P), S_{b \bar{b}}=0$, transitions above involve heavy-quark spin-flip, yet rates not suppressed, violating heavy-quark-spin conservation

- This contradiction is only apparent. Expressing the states $Z_{b}$ and $Z_{b}^{\prime}$ in the basis of definite $b \bar{b}$ and light quark $q \bar{q}$ spins

$$
\left|Z_{b}\right\rangle=\frac{\alpha\left|1_{q \bar{q}}, 0_{b \bar{b}}\right\rangle-\beta\left|0_{q \bar{q}}, 1_{b \bar{b}}\right\rangle}{\sqrt{2}},\left|Z_{b}^{\prime}\right\rangle=\frac{\beta\left|1_{q \bar{q}}, 0_{b \bar{b}}\right\rangle+\alpha\left|0_{q \bar{q}}, 1_{b \bar{b}}\right\rangle}{\sqrt{2}}
$$

and defining ( $g$ are the effective couplings at the vertices $Y Z_{b} \pi$ and $Z_{b} h_{b} \pi$ )

$$
\begin{aligned}
& g_{Z} \equiv g\left(Y \rightarrow Z_{b} \pi\right) g\left(Z_{b} \rightarrow h_{b} \pi\right) \propto-\alpha \beta\left\langle h_{b} \mid Z_{b}\right\rangle\left\langle Z_{b} \mid Y\right\rangle \\
& g_{Z^{\prime}} \equiv g\left(Y \rightarrow Z_{b}^{\prime} \pi\right) g\left(Z_{b}^{\prime} \rightarrow h_{b} \pi\right) \propto \alpha \beta\left\langle h_{b} \mid Z_{b}^{\prime}\right\rangle\left\langle Z_{b}^{\prime} \mid Y\right\rangle
\end{aligned}
$$


Heavy-Quark-Spin Flip in $\mathrm{Y}(10890) \rightarrow Z_{b} / Z_{b}^{\prime}+\pi \rightarrow h_{b}(1 P, 2 P) \pi \pi$

- Within errors, Belle data is consistent with heavy quark spin conservation, which requires $g_{Z}=-g_{Z^{\prime}}$

To determine the coefficients $\alpha$ and $\beta$, one has to resort to $s_{b \bar{b}}: 1 \rightarrow 1$ transitions

$$
\mathrm{Y}(10890) \rightarrow Z_{b} / Z_{b}^{\prime}+\pi \rightarrow \mathrm{Y}(n S) \pi \pi(n=1,2,3)
$$

The analogous effective couplings are

$$
\begin{aligned}
& f_{Z}=f\left(Y \rightarrow Z_{b} \pi\right) f\left(Z_{b} \rightarrow Y(n S) \pi\right) \propto|\beta|^{2}\left\langle Y(n S) \mid 0_{q \bar{q}}, 1_{b \bar{b}}\right\rangle\left\langle 0_{q \bar{q}}, 1_{b \bar{b}} \mid \mathrm{Y}\right\rangle \\
& f_{Z^{\prime}}=f\left(Y \rightarrow Z_{b}^{\prime} \pi\right) f\left(Z_{b}^{\prime} \rightarrow Y(n S) \pi\right) \propto|\alpha|^{2}\left\langle Y(n S) \mid 0_{q \bar{q}}, 1_{b \bar{b}}\right\rangle\left\langle 0_{q \bar{q}}, 1_{b \bar{b}} \mid Y\right\rangle
\end{aligned}
$$

- Dalitz analysis indicates:

$\mathrm{Y}(10890) \rightarrow Z_{b} / Z_{b}^{\prime}+\pi \rightarrow \mathrm{Y}(n S) \pi \pi(n=1,2,3)$ proceed mainly through the resonances $Z_{b}$ and $Z_{b}^{\prime}$, though $Y(10890) \rightarrow Y(1 S) \pi \pi$ has a significant direct component, expected in tetraquark interpretation of $Y(10890)$ [A.A., S. Mishima, C. Hambrock, PRL 106, 092002 (2011)] 
Determination of $\alpha / \beta$ from $Y(10890) \rightarrow Z_{b} / Z_{b}^{\prime}+\pi \rightarrow Y(n S) \pi \pi(n=1,2,3)$

- A comprehensive analysis of the Belle data including the direct and resonant components is required to test the underlying dynamics, yet to be carried out

- Parametrizing the amplitudes in terms of two Breit-Wigners, one can determine the ratio $\alpha / \beta$

$$
\begin{gathered}
s_{b \bar{b}}: 1 \rightarrow 1 \text { transition }: \\
\overline{\text { Rel.Norm. }}=0.85 \pm 0.08=|\alpha|^{2} /|\beta|^{2} \\
\overline{\text { Rel.Phase }}=(-8 \pm 10)^{\circ} \\
s_{b \bar{b}}: 1 \rightarrow 0 \text { transition }: \\
\overline{\text { Rel.Norm. }}=1.4 \pm 0.3 \\
\overline{\text { Rel.Phase }}=(185 \pm 42)^{\circ}
\end{gathered}
$$

Within errors, the tetraquark assignment with $\alpha=\beta=1$ is supported, i.e.,

$$
\begin{gathered}
\left|Z_{b}\right\rangle=\frac{\left|1_{b q}, 0_{\bar{b} \bar{q}}\right\rangle-\left|0_{b q}, 1_{\bar{b} \bar{q}}\right\rangle}{\sqrt{2}},\left|Z_{b}^{\prime}\right\rangle=\left|1_{b q}, 1_{\bar{b} \bar{q}}\right\rangle_{J=1} \\
\left|Z_{b}\right\rangle=\frac{\left|1_{q \bar{q}}, 0_{b \bar{b}}\right\rangle-\left|0_{q \bar{q}}, 1_{b \bar{b}}\right\rangle}{\sqrt{2}},\left|Z_{b}^{\prime}\right\rangle=\frac{\left|1_{q \bar{q}}, 0_{b \bar{b}}\right\rangle+\left|0_{q \bar{q}}, 1_{b \bar{b}}\right\rangle}{\sqrt{2}}
\end{gathered}
$$


Tetraquark model for $Y_{b}$ production and decays

- Van Royen-Weisskopf formula $\Rightarrow \Gamma\left(1^{--} \rightarrow e^{+} e^{-}\right)$

Assumption: Point-like diquarks [AA, Hambrock, Mishima, PRL 106 (2011), 092002]

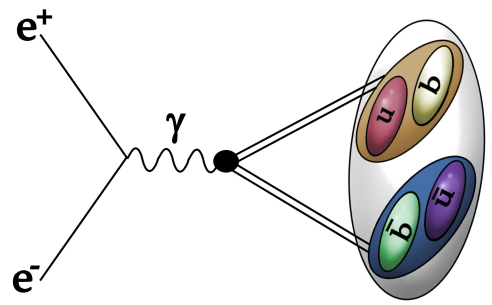


Tetraquark model for $Y_{b}$ production and decays

- Van Royen-Weisskopf formula $\Rightarrow \Gamma\left(1^{--} \rightarrow e^{+} e^{-}\right)$

Assumption: Point-like diquarks [AA, Hambrock, Mishima, PRL 106 (2011), 092002]

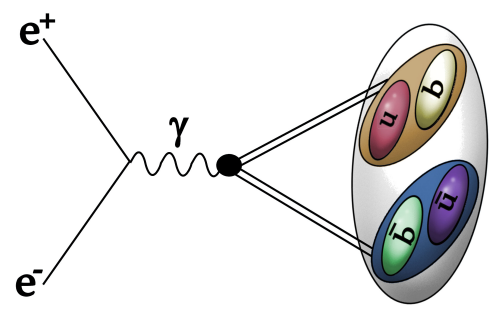

$$
\Gamma_{e e}\left(Y_{[b, l / h]}\right)=\frac{24 \alpha^{2} Q_{[b, l / h]}^{2}}{M_{Y_{[b, l / h]}}^{4}} \kappa^{2}\left|R_{11}^{(1)}(0)\right|^{2}
$$


Tetraquark model for $Y_{b}$ production and decays

- Van Royen-Weisskopf formula $\Rightarrow \Gamma\left(1^{--} \rightarrow e^{+} e^{-}\right)$

Assumption: Point-like diquarks [AA, Hambrock, Mishima, PRL 106 (2011), 092002]

$$
\Gamma_{e e}\left(Y_{[b, l / h]}\right)=\frac{24 \alpha^{2} Q_{[b, l / h]}^{2}}{M_{Y_{[b, l / h]}}^{4}} \kappa^{2}\left|R_{11}^{(1)}(0)\right|^{2}
$$


Tetraquark model for $Y_{b}$ production and decays

- Van Royen-Weisskopf formula $\Rightarrow \Gamma\left(1^{--} \rightarrow e^{+} e^{-}\right)$

Assumption: Point-like diquarks [AA, Hambrock, Mishima, PRL 106 (2011), 092002]

$$
\begin{aligned}
& \text { radial tetraquark WF @ origin } \\
& \Gamma_{e e}\left(Y_{[b, l / h]}\right)=\frac{24 \alpha^{2} Q_{[b, l / h]}^{2}}{M_{Y_{[b, l / h]}^{4}}^{4}} \kappa^{2}\left|R_{11}^{(\text {(4) }}(0)\right|^{2}
\end{aligned}
$$


Tetraquark model for $Y_{b}$ production and decays

- Van Royen-Weisskopf formula $\Rightarrow \Gamma\left(1^{--} \rightarrow e^{+} e^{-}\right)$

Assumption: Point-like diquarks [AA, Hambrock, Mishima, PRL 106 (2011), 092002]

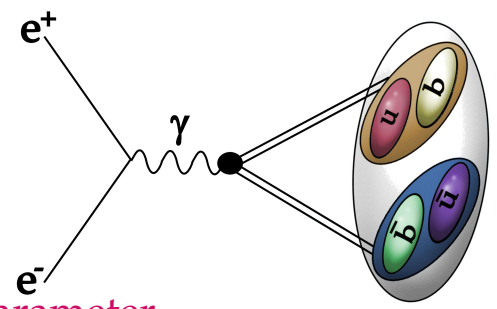

hadronic size parameter

$$
\Gamma_{e e}\left(Y_{[b, l / h]}\right)=\frac{24 \alpha^{2} Q_{[b, l / h]}^{2}}{M_{Y_{[b, l / h]}}^{4}} \kappa^{2}\left|R_{11}^{(1)}(0)\right|^{2}
$$


Tetraquark model for $Y_{b}$ production and decays

- Van Royen-Weisskopf formula $\Rightarrow \Gamma\left(1^{--} \rightarrow e^{+} e^{-}\right)$

Assumption: Point-like diquarks [AA, Hambrock, Mishima, PRL 106 (2011), 092002]

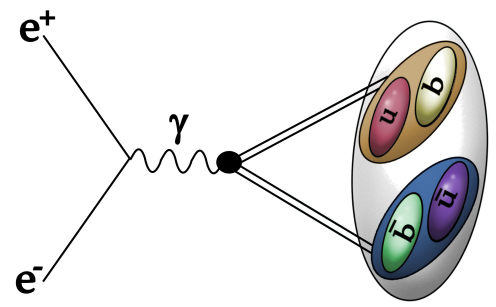

$$
\Gamma_{e e}\left(Y_{[b, l / h]}\right)=\frac{24 \alpha^{2} Q_{[b, l / h]}^{2}}{M_{Y_{[b, l / h]}}^{4}} \kappa^{2}\left|R_{11}^{(1)}(0)\right|^{2}
$$

Suppressed $\mathcal{O}(10)$ vs $\mathrm{Y}(5 S)$

Production ratio: $\Gamma_{Y_{[b, l]}} / \Gamma_{Y_{[b, h]}}=\left(\frac{1-2 \tan \theta}{2+\tan \theta}\right)^{2}$

Isospin breaking through production

$$
\text { e.g. } \frac{\sigma_{Y(1 S) K^{+} K^{-}}}{\sigma_{Y(1 S) K^{0} K^{0}}}=\frac{Q_{[b u]}^{2}}{Q_{[b d]}^{2}}=\frac{1}{4}
$$


Continuum

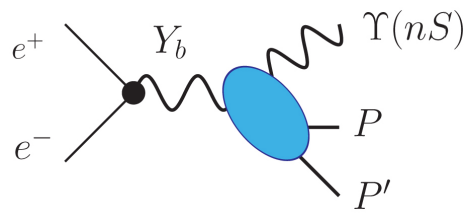

Resonance

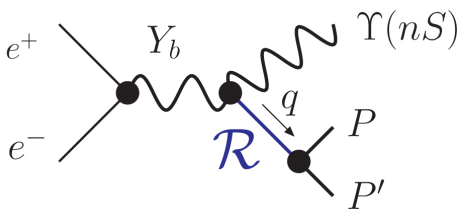

- Breit-Wigner shape for resonance:

$$
\frac{1}{\left(q^{2}-M^{2}\right)+i M \Gamma}
$$

$q^{2} \equiv M_{P P^{\prime}}^{2} \rightarrow$ Resonances show in $M_{P P^{\prime}}$ spectrum

Not in $M_{Y P}$ spectrum since $Z_{b}$ negligible 
Fit to $\sigma\left(e^{+} e^{-} \rightarrow Y_{b} \rightarrow \mathrm{Y}(1 S) \pi^{+} \pi^{-}\right)$

[AA, Hambrock, Jamil Aslam; PRL 104 (2010) 162001

AA, Hambrock, Mishima, PRL 106 (2011), 092002]

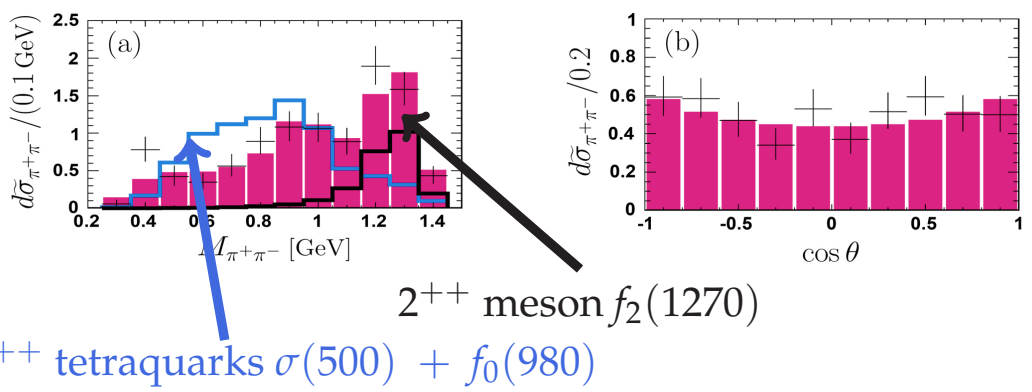

- Fit results, data from [Belle, PRL 08]

- $\chi^{2} /$ d.o.f. $=21.5 / 15 \rightarrow$ Good agreement with data

- Clear resonance dominance! 
Predictions for $\mathrm{Y}(1 S)\left(K^{+} K^{-}, \eta \pi^{0}\right)$

[AA, Hambrock, Mishima, PRL 106 (2011), 092002]

Fit determines couplings (assume $S U(3)$ flavor symmetry for couplings $\left(\sigma(500), f_{0}(980), a_{0}(980)\right) \rightarrow P P^{\prime}$, ['t Hooft, Isidori, Maiani, Polosa, Riquer, PLB 08] $)$

predictions for spectra:
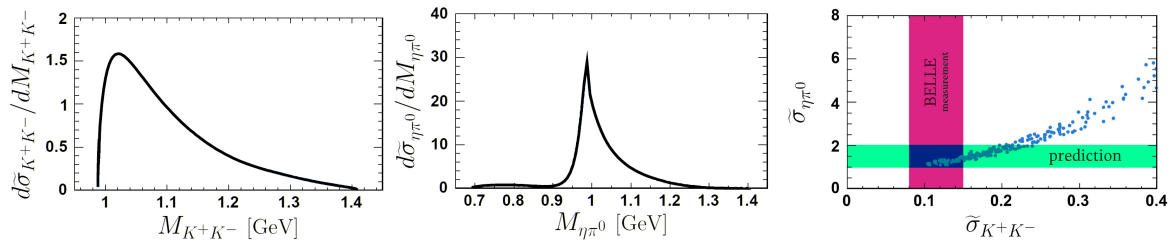

- Agreement with $\widetilde{\sigma}_{K^{+} K^{-}}=0.11_{-0.03}^{+0.04}$ (BELLE)

$\rightarrow 1.0 \lesssim \widetilde{\sigma}_{\eta \pi^{0}} \lesssim 2.0$ predicted

- Resonance dominance

Characteristic shape

Good tests (relying on $Y_{b}$ has 2 flavor states) 


\section{Are $Y_{c}, Z_{c} s, Y_{b}$ and $Z_{b}$ s related?}

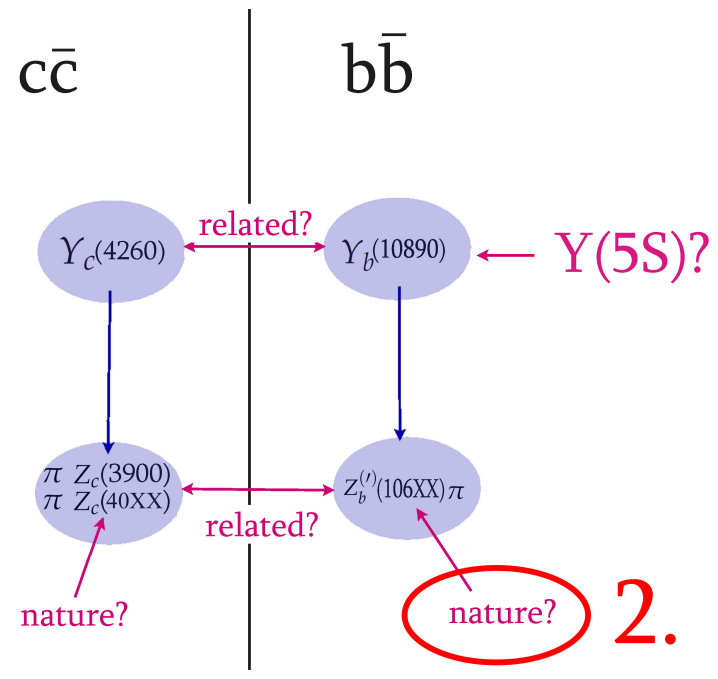




\section{Pentaquarks}

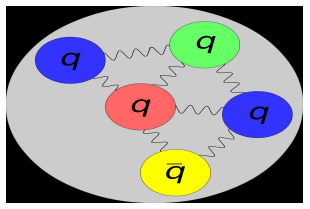

- Pentaquarks remained cursed under the shadow of the botched discoveries of $\Theta(1540), \Phi(1860), \Theta_{c}(3100)$ !

- Review on Pentaquarks [C.G. Wohl in PDG (2014)]:

There are two or three recent experiments that find weak evidence for signals near the nominal masses, but there is simply no point in tabulating them in view of the overwhelming evidence that the claimed pentaquarks do not exist. The only advance in particle physics thought worthy of mention in the American Institute of Physics "Physics News in 2003" was a false alarm. The whole story - is a curious episode in the history of science. 
Elusive Pentaquark Comes into View! (R. Aaij et al., PRL 115, 072001 (2015)

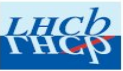

CERN-PH-EP-2015-153

LHCb-PAPER-2015-029

July 13,2015

\title{
Observation of $J / \psi p$ resonances consistent with pentaquark states in $\boldsymbol{\Lambda}_{b}^{0} \rightarrow J / \psi \boldsymbol{K}^{-} \boldsymbol{p}$ decays
}

The LHCb collaboration [1]

\begin{abstract}
Observations of exotic structures in the $J / \psi p$ channel, which we refer to as charmonium-pentaquark states, in $\Lambda_{b}^{0} \rightarrow J / \psi K^{-} p$ decays are presented. The data sample corresponds to an integrated luminosity of $3 \mathrm{fb}^{-1}$ acquired with the LHCb detector from 7 and $8 \mathrm{TeV} p p$ collisions. An amplitude analysis of the three-body final-state reproduces the two-body mass and angular distributions. To obtain a satisfactory fit of the structures seen in the $J / \psi p$ mass spectrum, it is necessary to include two Breit-Wigner amplitudes that each describe a resonant state. The significance of each of these resonances is more than 9 standard deviations. One has a mass of $4380 \pm 8 \pm 29 \mathrm{MeV}$ and a width of $205 \pm 18 \pm 86 \mathrm{MeV}$, while the second is narrower, with a mass of $4449.8 \pm 1.7 \pm 2.5 \mathrm{MeV}$ and a width of $39 \pm 5 \pm 19 \mathrm{MeV}$. The preferred $J^{P}$ assignments are of opposite parity, with one state having spin $3 / 2$ and the other $5 / 2$.
\end{abstract}


The Pentaquarks $P_{c}^{+}(4380)$ and $P_{c}^{+}(4450)$ as resonant $J / \psi p$ states

- Discovery Channel ( $\left.\mathrm{LHC} ; \sqrt{s}=7 \& 8 \mathrm{TeV} ; \int L d t=3 \mathrm{fb}^{-1}\right)$

$$
p p \rightarrow b \bar{b} \rightarrow \Lambda_{b} X ; \quad \Lambda_{b} \rightarrow K^{-} J / \psi p
$$
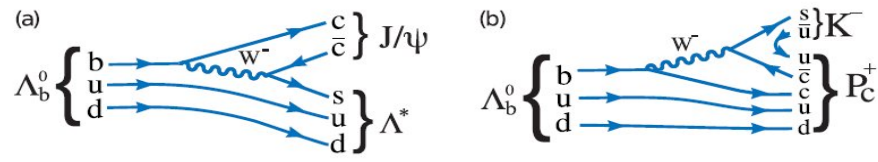

Figure 1: Feynman diagrams for (a) $\Lambda_{b}^{0} \rightarrow J / \psi \Lambda^{*}$ and (b) $\Lambda_{b}^{0} \rightarrow P_{c}^{+} K^{-}$decay.
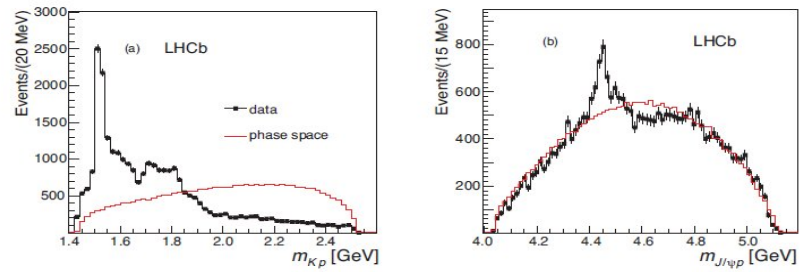

Figure 2: Invariant mass of (a) $K^{-} p$ and (b) $J / \psi p$ combinations from $\Lambda_{b}^{0} \rightarrow J / \psi K^{-} p$ decays. The solid (red) curve is the expectation from phase space. The background has been subtracted. 
Model fits with two $\left[P_{\mathcal{C}}^{+}(4380)\right.$ and $\left.P_{\mathcal{C}}^{+}(4450)\right]$ states

- Fits with two $P_{c}^{+}$states. Acceptable fits found for several $J^{P}$ combinations

- The best fit yields $J^{P}=\left(3 / 2^{-}, 5 / 2^{+}\right)$for $\left[P_{c}^{+}(4380), P_{c}^{+}(4450)\right]$ states. Both the $m_{K p}$ and $m_{J / \psi p}$ projections are well described
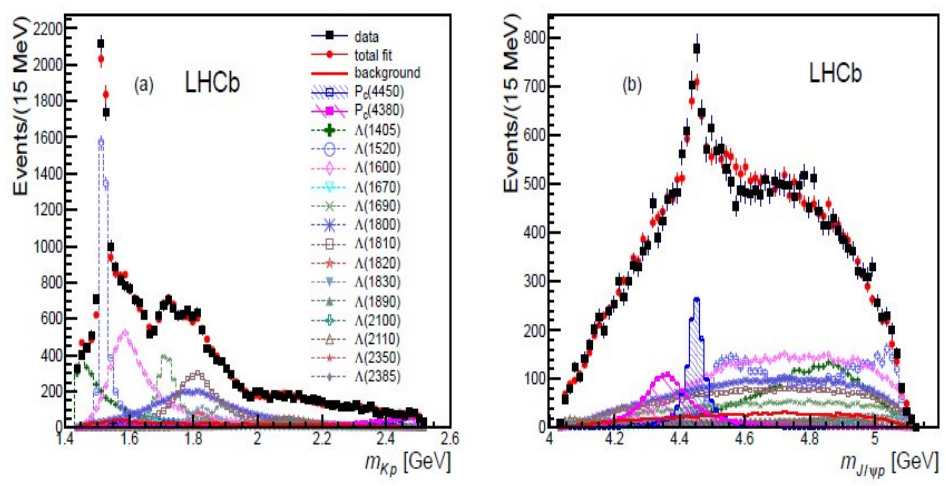
Summary of the LHCb Pentaquark Measurements

- Higher mass state (statistical significance $12 \sigma$ )

$$
M=4449.8 \pm 1.7 \pm 2.5 \mathrm{MeV} ; \quad \Gamma=39 \pm 5 \pm 19 \mathrm{MeV}
$$

- Lower mass state (statistical significance $9 \sigma$ )

$$
M=4380 \pm 8 \pm 29 \mathrm{MeV} ; \Gamma=205 \pm 18 \pm 86 \mathrm{MeV}
$$

- Fitted Values of the real and imaginary parts of the amplitudes

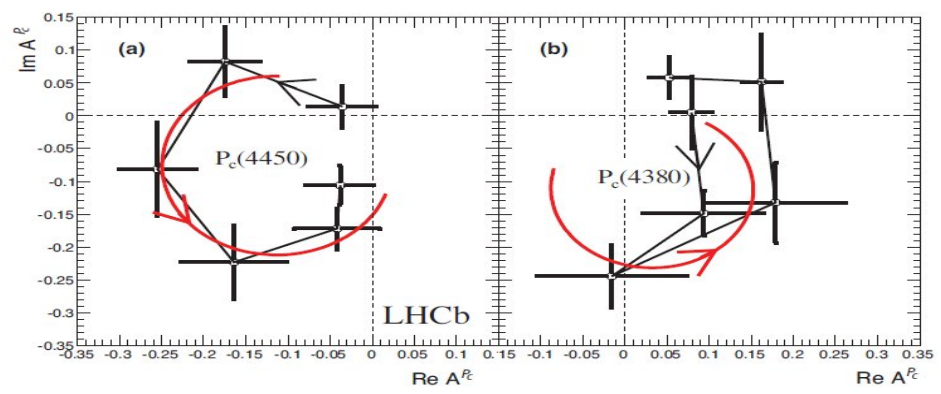

- For $P_{c}^{+}(4450)$, fit shows a phase change in amplitudes consistent with a resonance 
Summary of the LHCb Pentaquark Measurements (Contd.)

Possible $J^{P}$ assignments and the energies of the nearby thresholds

\begin{tabular}{lll}
\hline & $P_{c}(4380)^{+}$ & $P_{c}(4450)^{+}$ \\
\hline Mass & $4380 \pm 8 \pm 29$ & $4449.8 \pm 1.7 \pm 2.5$ \\
Width & $205 \pm 18 \pm 86$ & $35 \pm 5 \pm 19$ \\
\hline Assignment 1 & $3 / 2^{-}$ & $5 / 2^{+}$ \\
Assignment 2 & $3 / 2^{+}$ & $5 / 2^{-}$ \\
Assignment 3 & $5 / 2^{+}$ & $3 / 2^{-}$ \\
\hline$\Sigma_{c}^{*+} \bar{D}^{0}$ & $4382.3 \pm 2.4$ & \\
$\chi_{c 1} p$ & & $4448.93 \pm 0.07$ \\
$\Lambda_{c}^{*+} \bar{D}^{0}$ & & $4457.09 \pm 0.35$ \\
$\Sigma_{c}^{+} \bar{D}^{* 0}$ & & $4459.9 \pm 0.5$ \\
$\Sigma_{c}^{+} \bar{D}^{0} \pi^{0}$ & & $4452.7 \pm 0.5$ \\
\hline
\end{tabular}


Theoretical Interpretations of the LHCb Pentaquarks

\section{Rescattering-induced kinematic effects}

- Feng-Kun Guo, Ulf-G.Meißner, Wei Wang, Zhi Yang, arxiv:1507.04950

- Xiao-Hai Liu, Qian Wang, Qiang Zhao, arxiv:1507.05359

- M. Mikhasenko, arxiv:1507.06552

- Ulf-G.Meißner, Jose A. Oller, arxiv:1507.07478

\section{Open-charm-baryon and -meson bound states}

- Hua-Xing Chen, Wei Chen, Xiang Liu, T.G. Steele, Shi-Lin Zhu, arxiv:1507.03717

- Jun He, arxiv:1507.05200

L. Roca, J. Nieves, E. Oset, arxiv:1507.04249

- Rui Chen, Xiang-Liu, arxiv:1507.03704

- C. W. Xiao and Ulf-G.Meißner, arxiv:1508.00924

\section{Pentaquarks as Baryocharmonia}

- Formation of hidden-charm pentaquarks in photon-nucleon collisions V. Kubarovsky and M.B. Voloshin, arxiv:1508.00888 
Theoretical Interpretations of the LHCb Pentaquarks (Contd.)

\section{Compact Pentaquarks}

[. Maiani, A.D. Polosa, V. Riquer, arxiv: 1507.04980

- Richard F. Lebed, arxiv:1507.05867

- Guan-Nan Li, Xiao-Gang He, Min He, arxiv:1507.08252

- A. Mironov, A. Morozov, arxiv:1507.04694

A.V. Anisovich et al., arxiv:1507.07652

R. Ghosh, A. Bhattacharya, B. Chakrabarti, arxiv:1508.00356

- Zhi-Gang Wang, arxiv:1508.01468

- Zhi-Gang Wang, Tao Huang, arxiv:1508.04189 
Pentaquarks as rescattering-induced kinematic effects

\section{[Feng-Kun Guo et al.; arxiv:1507.04950]}

- Hypothesis: Kinematic effects can result in a narrow structure around the $\chi_{c 1} p$ threshold in the $J / \psi p$ invariant mass of the decay $\Lambda_{b}^{0} \rightarrow K^{-} J / \psi p$ $M_{P_{c}(4450)}-M_{\chi_{c 1}}-M_{p}=(0.9 \pm 3.1) \mathrm{MeV}$

- Two possible mechanisms:

a) 2-point loop with a 3-body production $\Lambda_{b}^{0} \rightarrow K^{-} \chi_{c 1} p$ followed by the rescattering process $\chi_{c 1} p \rightarrow J / \psi p$

b) The $K^{-} p$ is produced from an intermediate $\Lambda^{*}$ and the proton rescatters with the $\chi_{c 1}$ into a $J / \psi p$

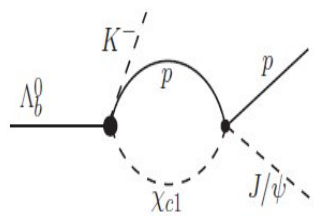

(a)

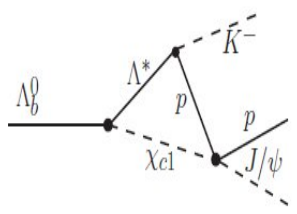

(b) 
Pentaquarks as rescattering-induced kinematic effects (Contd.)

[Feng-Kun Guo et al.; arxiv:1507.04950]

- Amplitude for Fig. (a) $\left(\mu=\right.$ reduced mass and $\left.f_{\Lambda}\left(\vec{q}^{2}\right)=\exp \left(-2 \vec{q}^{2} / \Lambda^{2}\right)\right)$

$$
G_{\Lambda}(E)=\int \frac{d^{3} q}{(2 \pi)^{3}} \frac{\vec{q}^{2} f_{\Lambda}\left(\vec{q}^{2}\right)}{E-m_{p}-m_{\chi_{c 1}}-\vec{q}^{2} /(2 \mu)}
$$

- Fitting the Argand diagram for $P_{c}(4450)$ with $\mathcal{A}_{(a)}=N\left(b+G_{\Lambda}(E)\right)$ determines the normalization $N$, the constant background $b$ and $\Lambda$

- Amplitude for Fig. (b) is assumed dominated by $\Lambda^{*}(1890)$-exchange, and its width is varied from $10 \mathrm{MeV}$ to $100 \mathrm{MeV}$, leading to sharp peaks at $\operatorname{Re} \sqrt{s}=4450 \mathrm{MeV}$
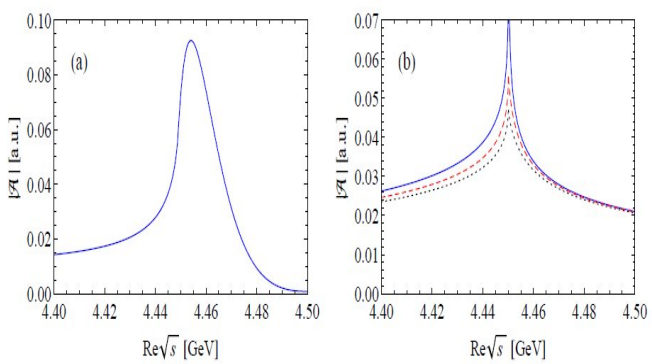
Pentaquarks as hadronic molecular states [Rui Chen et al., arxiv:1507.03704]

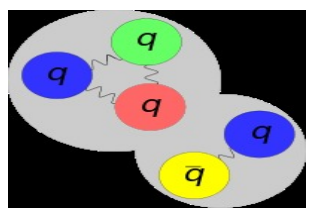

Identify $\mathbb{P}_{c}^{+}(4380)$ with $\Sigma_{c}(2455) \bar{D}^{*}$ and $\mathbb{P}_{c}^{+}$(4450) with $\Sigma_{c}(2520) \bar{D}^{*}$ bound by a pion exchange

- Effective Lagrangians:

$$
\begin{aligned}
& \mathcal{L}_{\mathcal{P}}=i g \operatorname{Tr}\left[\bar{H}_{a}^{(\bar{Q})} \gamma^{\mu} A_{a b}^{\mu} \gamma_{5} H_{b}^{(\bar{Q})}\right] \\
& \mathcal{L}_{\mathcal{S}}=-\frac{3}{2} g_{1} \epsilon^{\mu \lambda \nu \kappa_{\sigma_{\mathcal{K}}} \operatorname{Tr}}\left[\overline{\mathcal{S}}_{\mu} A_{\nu} \mathcal{S}_{\lambda}\right]
\end{aligned}
$$

- $H_{a}^{(\bar{Q})}=\left[P_{a}^{*(\bar{Q}) \mu} \gamma_{\mu}-P_{a}^{(\bar{Q})} \gamma_{5}\right](1-\not b) / 2 ; v=(0, \overrightarrow{1})$ is a pseudoscalar and vector charmed meson multiplet $\left(D, D^{*}\right)$; $\mathcal{S}_{\mu}=\sqrt{1 / 3}\left(\gamma_{\mu}+v_{\mu}\right) \gamma^{5} \mathcal{B}_{6}+\mathcal{B}_{6 \mu}^{*}$ stands for the charmed baryon multiplet, with $\mathcal{B}_{6}$ and $\mathcal{B}_{6 \mu}^{*}$ corresponding to the $J^{P}=1 / 2^{+}$and $J^{P}=3 / 2^{+}$in $6_{F}$ flavor representation;

$A_{\mu}$ is an axial-vector current, containing a pion chiral multiplet 
Eff. potentials, energy levels \& wave-functions of the $\Sigma_{c}^{(*)} \bar{D}^{*}$ systems
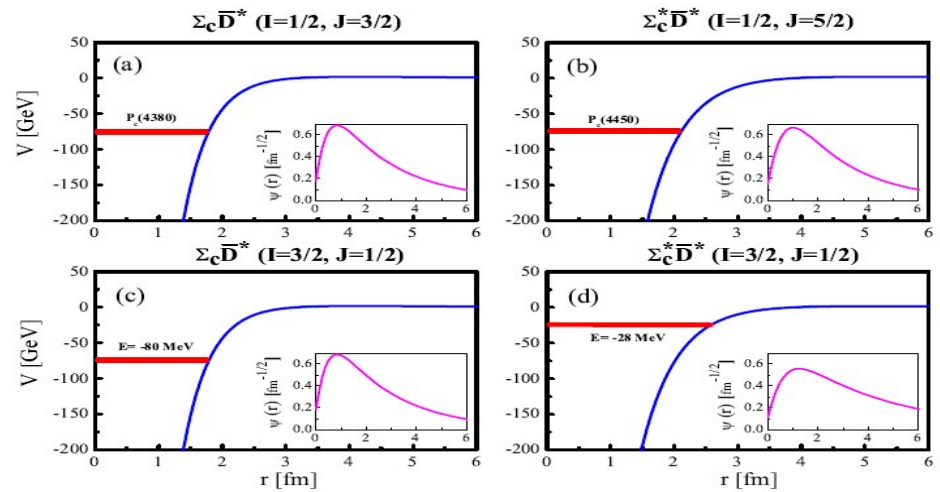

$\mathbb{P}_{c}(4380)$ is a $\Sigma_{c} \bar{D}^{*}(I=1 / 2, J=3 / 2)$ molecule $\mathbb{P}_{c}(4450)$ is a $\Sigma_{c}^{*} \bar{D}^{*}(I=1 / 2, J=5 / 2)$ molecule

- Predict two additional hidden-charm molecular pentaquark states, $\Sigma_{c} \bar{D}^{*}(I=3 / 2, J=1 / 2)$ and $\Sigma_{c}^{*} \bar{D}^{*}(I=3 / 2, J=1 / 2)$, which are isospin partners of $\mathbb{P}_{c}(4380)$ and $\mathbb{P}_{c}(4450)$, decaying into $\Delta(1232) J / \psi$ and $\Delta(1232) \eta_{c}$

- A rich pentaquark spectrum of states for the hidden-bottom $\left(\Sigma_{b} B^{*}, \Sigma_{b}^{*} B^{*}\right)$, $B_{c}$-like $\left(\Sigma_{c} B^{*}, \Sigma_{c}^{*} B^{*}\right)$ and $\left(\Sigma_{b} \bar{D}^{*}, \Sigma_{b}^{*} \bar{D}^{*}\right)$ with well-defined $(I, J)$ are predicted 


\section{Effective Hamiltonian for Pentaquarks}

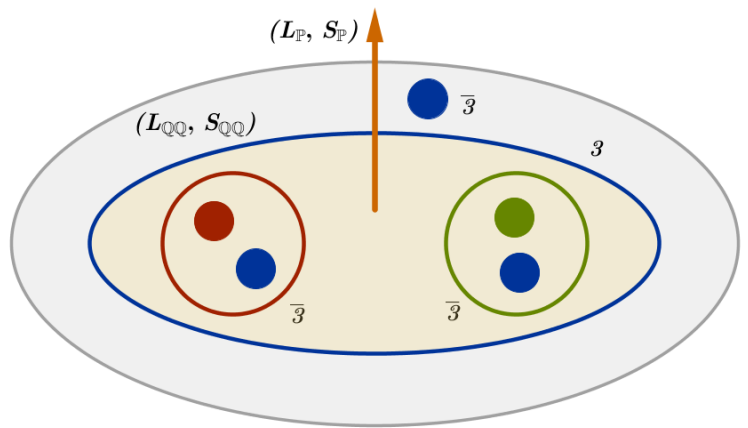

Diquark - Diquark - Antiquark Model of Pentaquarks

$$
H_{\mathrm{eff}}(\mathbb{P})=H_{\mathrm{eff}}([\mathcal{Q Q}])+m_{\bar{c}}+\kappa_{\bar{c}[\mathcal{Q Q}]}\left(s_{\bar{c}} \cdot S_{[\mathcal{Q Q}]}\right)-2 a_{\mathbb{P}}\left(L_{\mathbb{P}} \cdot S_{\mathbb{P}}\right)+\frac{B_{\mathbb{P}}}{2}\left\langle L_{\mathbb{P}}{ }^{2}\right\rangle
$$

- $S_{[\mathcal{Q Q}]}$ is the spin of the tetraquark; $s_{\bar{C}}$ is the spin of the $\bar{c}$ $L_{\mathbb{P}}$ and $S_{\mathbb{P}}$ are the orbital angular momentum and spin of the pentaquark, respectively 
Pentaquarks in the diquark model [Maiani et al., arxiv:1507.04980]

$\Lambda_{b}($ bud $) \rightarrow \mathbb{P}^{+} K^{-}$decaying according to $\mathbb{P}^{+} \rightarrow J / \Psi+p$

$\mathbb{P}^{+}$carry a unit of baryonic number and have the valence quarks

$$
\mathbb{P}^{+}=\bar{c} c u u d
$$

Assume the assignments

$$
\begin{aligned}
& \mathbb{P}^{+}\left(3 / 2^{-}\right)=\left\{\bar{c}[c q]_{s=1}\left[q^{\prime} q^{\prime \prime}\right]_{s=1}, L=0\right\} \\
& \mathbb{P}^{+}\left(5 / 2^{+}\right)=\left\{\bar{c}[c q]_{s=1}\left[q^{\prime} q^{\prime \prime}\right]_{s=0}, L=1\right\}
\end{aligned}
$$

Mass difference:

- Level spacing for $\Delta L=1$ in light baryons; $\Lambda(1405)-\Lambda(1116) \sim 290 \mathrm{MeV}$

- Light-light diquark mass difference for $\Delta S=1$ : $\left[q q^{\prime}\right]_{S=1}-\left[q q^{\prime}\right]_{S=0}=\Sigma_{c}(2455)-\Lambda_{c}(2286) \simeq 170 \mathrm{MeV}$

- Orbital gap $\mathbb{P}^{+}\left(3 / 2^{-}\right)-\mathbb{P}^{+}\left(5 / 2^{+}\right)$is thereby reduced to $120 \mathrm{MeV}$, more or less in agreement with data, $70 \mathrm{MeV}$ 
Pentaquark production mechanisms in $\Lambda_{b}^{0} \rightarrow K^{-} J / \psi p$

- Two possible mechanisms are proposed by Maiani et al.

- In the first, $b$-quark spin is shared between the $K^{-}$, and the $\bar{c}$ and $[c u]$ components, the final $[u d]$ diquark has spin-0, Fig. A

- In the second, the $[u d]$ diquark is formed from the original $d$ quark, and the $u$ quark from the vacuum $u \bar{u}$; angular momentum is shared among all components, and the diquark $[u d]$ may have both spins, $s=0,1$, Fig. B

Which of the two diagrams dominate is a dynamical question; semileptonic decays of $\Lambda_{b}$ hint that the mechanism in Fig. B is dynamically suppressed

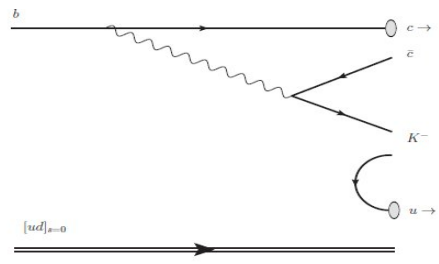

(A)

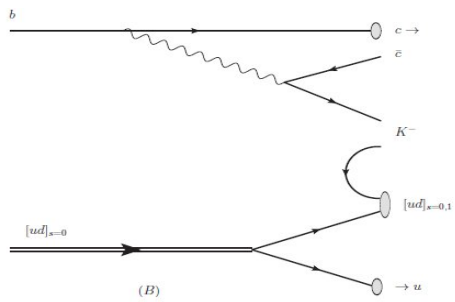


Flavor SU(3) structure of Pentaquarks

- Pentaquarks are of two types:

$$
\begin{aligned}
\mathbb{P}_{u} & =\epsilon^{\alpha \beta \gamma_{c_{\alpha}}}[c u]_{\beta, s=0,1}[u d]_{\gamma, s=0,1} \\
\mathbb{P}_{d} & =\epsilon^{\alpha \beta \gamma_{c_{\alpha}}}[c d]_{\beta, s=0,1}[u u]_{\gamma, s=1}
\end{aligned}
$$

- This leads to two distinct $S U(3)$ series of Pentaquarks

$$
\begin{aligned}
& \mathbb{P}_{A}=\epsilon^{\alpha \beta \gamma}\left\{\bar{c}_{\alpha}[c q]_{\beta, s=0,1}\left[q^{\prime} q^{\prime \prime}\right]_{\gamma, s=0}, L\right\}=\mathbf{3} \otimes \overline{\mathbf{3}}=\mathbf{1} \oplus \mathbf{8} \\
& \mathbb{P}_{S}=\epsilon^{\alpha \beta \gamma}\left\{\bar{c}_{\alpha}[c q]_{\beta, s=0,1}\left[q^{\prime} q^{\prime \prime}\right]_{\gamma, s=1}, L\right\}=\mathbf{3} \otimes \mathbf{6}=\mathbf{8} \oplus \mathbf{1 0}
\end{aligned}
$$

- For $S$ waves, the first and the second series have the angular momenta (multipicity)

$$
\begin{aligned}
& \mathbb{P}_{A}(L=0): \quad J=1 / 2(2), 3 / 2(1) \\
& \mathbb{P}_{S}(L=0): \quad J=1 / 2(3), 3 / 2(3), 5 / 2(1)
\end{aligned}
$$

- Maiani et al. propose to assign $\mathbb{P}\left(3 / 2^{-}\right)$to the $\mathbb{P}_{A}$ and $\mathbb{P}\left(5 / 2^{+}\right)$to the $\mathbb{P}_{S}$ series of Pentaquarks 
SU(3) based analysis of $\Lambda_{b} \rightarrow \mathbb{P}^{+} K^{-} \rightarrow(J / \psi p) K^{-}$

With respect to flavor $\operatorname{SU}(3), \Lambda_{b}(b u d) \sim \overline{3}$, and is isosinglet $I=0$

The weak non-leptonic Hamiltonian for $b \rightarrow c \bar{c} s$ decays is:

$$
H_{\mathrm{W}}^{(3)}(\Delta I=0, \Delta S=-1)
$$

With $M$ a nonet of $S U(3)$ light mesons, $\left\langle\mathbb{P}, M\left|H_{W}\right| \Lambda_{b}\right\rangle$ requires $\mathbb{P}+M$ to be in $8 \oplus 1$ representation

Recalling the $S U(3)$ group multiplication rule

$$
\begin{aligned}
8 \otimes 8 & =1 \oplus 8 \oplus 8 \oplus 10 \oplus \overline{10} \oplus 27 \\
8 \otimes 10 & =8 \oplus 10 \oplus 27 \oplus 35
\end{aligned}
$$

the decay $\left\langle\mathbb{P}, M\left|H_{\mathrm{W}}\right| \Lambda_{b}\right\rangle$ can be realized with $\mathbb{P}$ in either an octet (8) or a decuplet (10)

The discovery channel $\Lambda_{b} \rightarrow \mathbb{P}^{+} K^{-} \rightarrow J / \psi p K^{-}$corresponds to $\mathbb{P}$ in an octet (8) 


\section{Weak decays with $\mathbb{P}$ in Decuplet representation}

- Decays involving the decuplet (10) pentaquarks may also occur, if the light diquark pair having spin- $0[u d]_{s=0}$ in $\Lambda_{b}$ gets broken to produce a spin- 1 light diquark $[u d]_{s=1}$

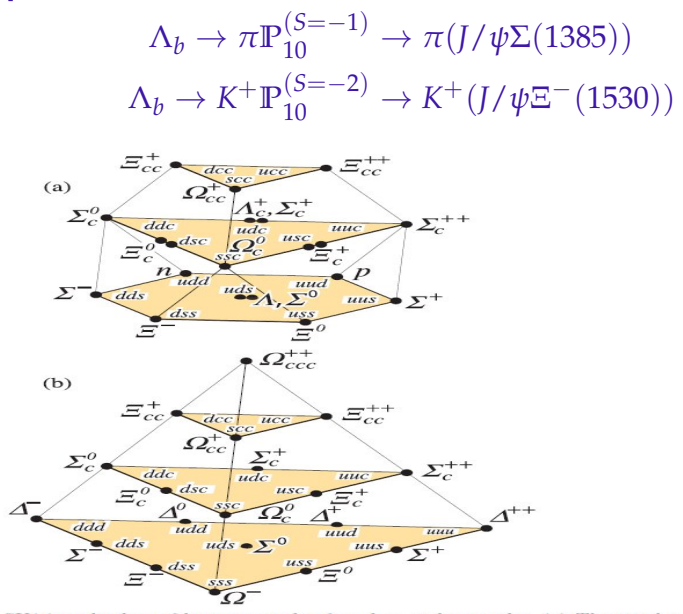

Figure 15.4: SU(4) multiplets of baryons made of $u, d, s$, and $c$ quarks. (a) The 20-plet with an SU(3) octet. (b) The 20-plet with an SU(3) decuplet. 
Weak decays with $\mathbb{P}$ in Decuplet representation - Contd.

Apart from $\Lambda_{b}(b u d)$, several $b$-baryons, such as $\Xi_{b}^{0}(u s b), \Xi_{b}^{-}(d s b)$ and $\Omega_{b}^{-}(s s b)$ undergo weak decays

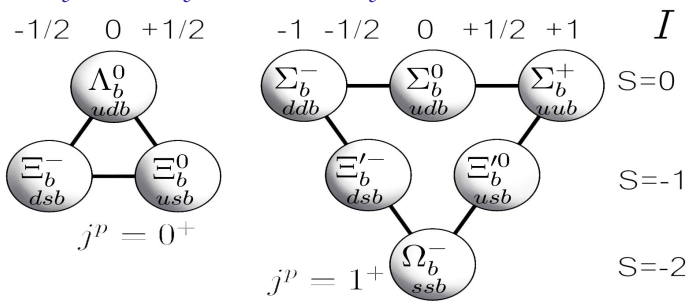

Examples of bottom-strange b-baryon in various charge combinations, respecting $\Delta I=0, \Delta S=-1$ are:

$$
\Xi_{b}^{0}(5794) \rightarrow K(J / \psi \Sigma(1385))
$$

which corresponds to the formation of the pentaquarks with the spin configuration $\left(q, q^{\prime}=u, d\right)$

$$
\mathbb{P}_{10}\left(\bar{c}[c q]_{s=0,1}\left[q^{\prime} s\right]_{s=0,1}\right)
$$


Weak decays with $\mathbb{P}$ in Decuplet representation - Contd.

The $s \bar{s}$ pair in $\Omega_{b}$ is in the symmetric (6) representation of flavor $S U(3)$ with spin 1 ; expected to produce decuplet Pentaquarks in association with a $\phi$ or a Kaon

$$
\begin{aligned}
& \Omega_{b}(6049) \rightarrow \phi\left(J / \psi \Omega^{-}(1672)\right) \\
& \Omega_{b}(6049) \rightarrow K(J / \psi \Xi(1387))
\end{aligned}
$$

These correspond, respectively, to the formation of the following pentaquarks $(q=u, d)$

$$
\begin{aligned}
& \mathbb{P}_{10}^{-}\left(\bar{c}[c s]_{s=0,1}[s s]_{s=1}\right) \\
& \mathbb{P}_{10}\left(\bar{c}[c q]_{s=0,1}[s s]_{s=1}\right)
\end{aligned}
$$

- These transitions are on firmer theoretical footings, as the initial [ss] diquark in $\Omega_{b}$ is left unbroken; more transitions can be found relaxing this condition 


\section{Summary}

- A new facet of $\mathrm{QCD}$ is opened by the discovery of the exotic $X, Y, Z$, and the pentaquark states $\mathbb{P}(4380)$ and $\mathbb{P}(4450)$

- Dedicated studies required to establish the nature of exotics in experiments and QCD

Important puzzles remain in the complex:

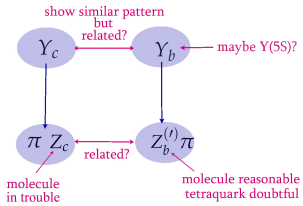

What is the nature of $Y_{c}(4260)$ ? A tetraquark? or a $c \bar{c} g$ hybrid?

What exactly is $Y(10888)$ ? Is it just $Y(5 S)$ ? Does $Y_{b}(10890)$ still exist?

- Line shape of multiquark resonances, such as $X(3872)$ and $\mathbb{P}(4450)$ can be measured at $\overline{\mathrm{P}} A N D A$, which will help in understanding the dynamics

We look forward to decisive experimental results from Belle-II, LHC and $\overline{\mathrm{P}} A N D A$ 


\section{Backup Slides}




\section{Hadroproduction of Bottomonia \& Exotic States}

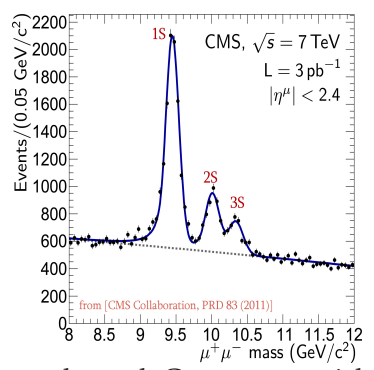

- $\mu^{+} \mu^{-}$channel: Common particle detection for bottomonia 


\section{Hadroproduction of Bottomonia \& Exotic States}

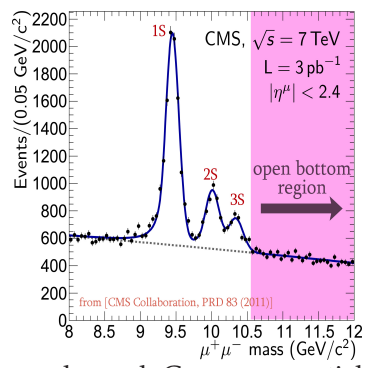

- $\mu^{+} \mu^{-}$channel: Common particle detection for bottomonia

- Above threshold difficult (BR $\left(\mu^{+} \mu^{-}\right)$drops)

present research focused on $1 S, 2 S, 3 S$ 


\section{Hadroproduction of Bottomonia \& Exotic States}
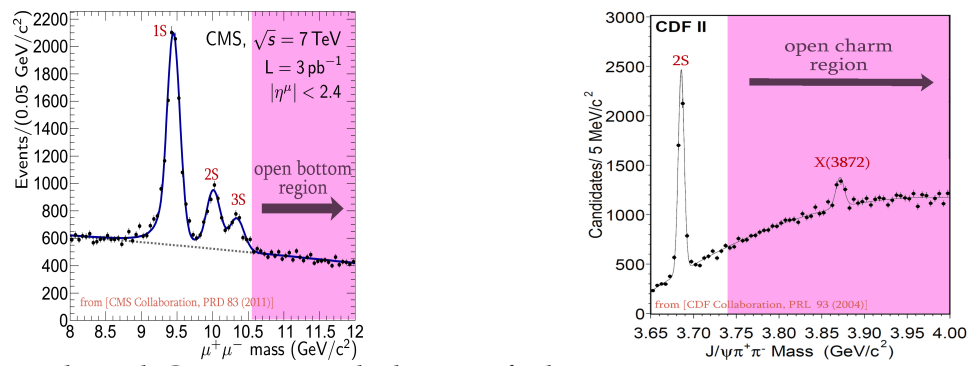

- $\mu^{+} \mu^{-}$channel: Common particle detection for bottomonia

- Above threshold difficult (BR $\left(\mu^{+} \mu^{-}\right)$drops)

$$
\text { present research focused on } 1 S, 2 S, 3 S
$$

Different final states (e.g. $\mu^{+} \mu^{-} \pi^{+} \pi^{-}$) allow for exotic searches 


\section{Hadroproduction of Bottomonia \& Exotic States}
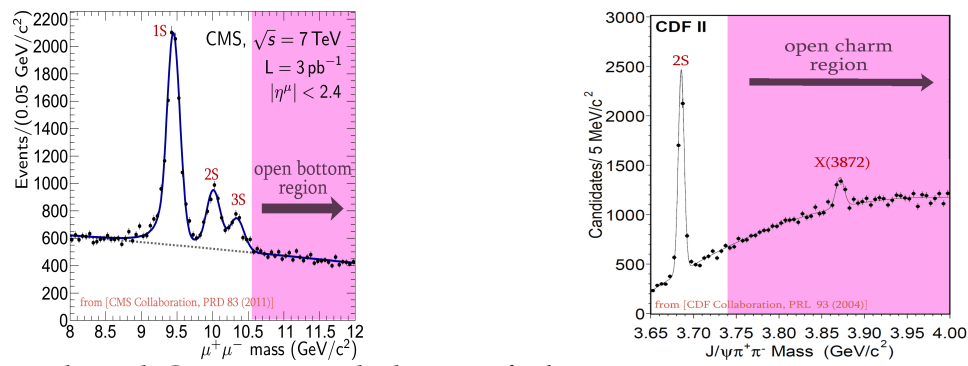

- $\mu^{+} \mu^{-}$channel: Common particle detection for bottomonia

- Above threshold difficult $\left(\operatorname{BR}\left(\mu^{+} \mu^{-}\right)\right.$drops)

$$
\text { present research focused on } 1 S, 2 S, 3 S
$$

- Different final states (e.g. $\mu^{+} \mu^{-} \pi^{+} \pi^{-}$) allow for exotic searches

[Ali, Hambrock, Wang, PRD 13] :

Acquire knowledge of bottomonia above hadronic thresholds (NRQCD, pNRQCD [Brambilla, Pineda, Soto, Vairo, NP 00] )

\section{clarify nature of observed states}




\section{NRQCD Framework}

$$
\sigma_{N}(p \bar{p}(p) \rightarrow \mathrm{Y}+\mathrm{X})
$$




\section{NRQCD Framework}

$$
\sigma_{N}(p \bar{p}(p) \rightarrow \mathrm{Y}+\mathrm{X})=\int d x_{1} d x_{2} \sum_{i, j} f_{i}\left(x_{1}\right) f_{j}\left(x_{2}\right)
$$




\section{NRQCD Framework}

$$
\sigma_{N}(p \bar{p}(p) \rightarrow \mathrm{Y}+\mathrm{X})=\int d x_{1} d x_{2} \sum_{i, j} f_{i}\left(x_{1}\right) f_{j}\left(x_{2}\right) \times \hat{\sigma}(i j \rightarrow \bar{b} b+X)
$$

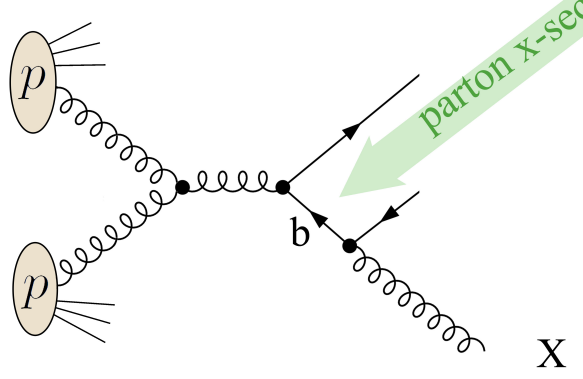




\section{NRQCD Framework}

$$
\sigma_{N}(p \bar{p}(p) \rightarrow \mathrm{Y}+\mathrm{X})=\int d x_{1} d x_{2} \sum_{i, j} f_{i}\left(x_{1}\right) f_{j}\left(x_{2}\right) \times \hat{\sigma}\left(i j \rightarrow\langle\bar{b} b\rangle_{N}+X\right)
$$

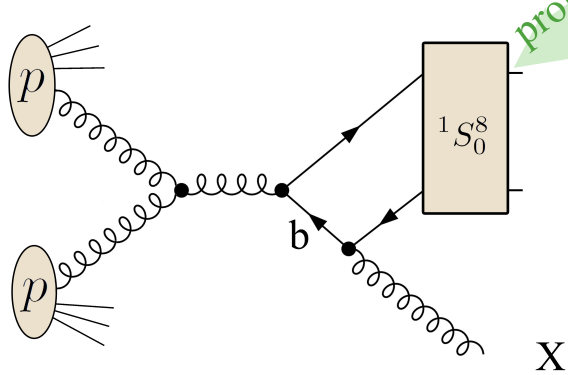




\section{NRQCD Framework}

$$
\sigma_{N}(p \bar{p}(p) \rightarrow \mathrm{Y}+\mathrm{X})=\int d x_{1} d x_{2} \sum_{i, j} f_{i}\left(x_{1}\right) f_{j}\left(x_{2}\right) \times \hat{\sigma}\left(i j \rightarrow\langle\bar{b} b\rangle_{N}+X\right)\langle\mathrm{O}[N]\rangle
$$

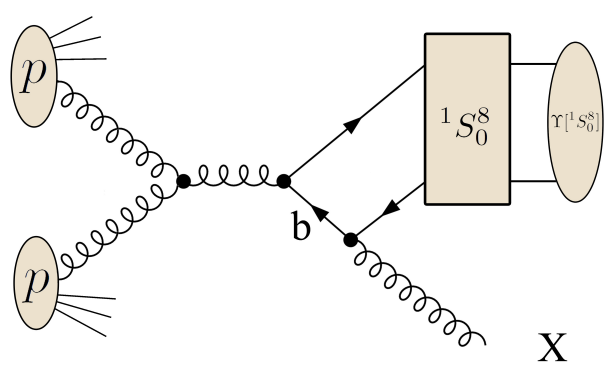




\section{NRQCD Framework}

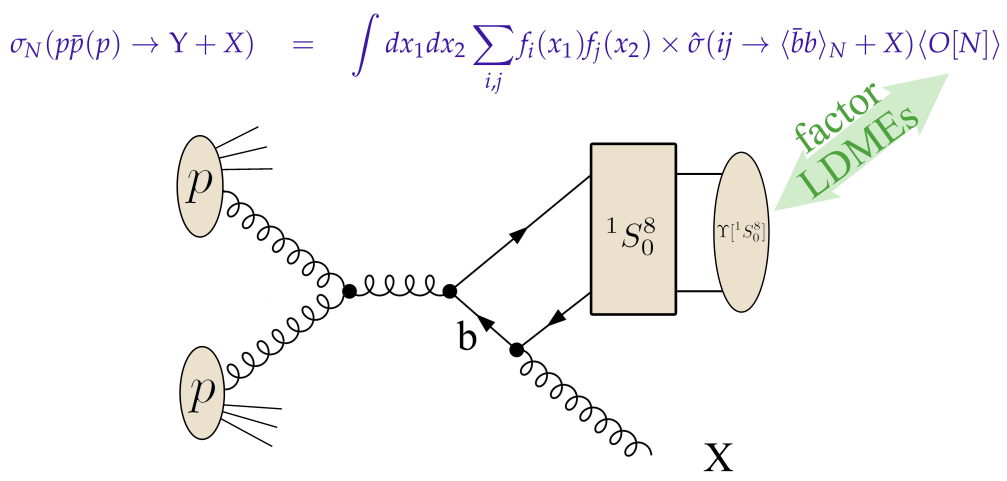

- partonic channels (include NLO for CS):

$\begin{array}{ll}g g \rightarrow \mathrm{Y}\left[{ }^{3} S_{1}^{1}\right]+g, & g g \rightarrow \mathrm{Y}\left[{ }^{1} S_{0}^{8},{ }^{3} S_{1}^{8}\right]+g \\ g q \rightarrow \mathrm{Y}\left[{ }^{1} S_{0}^{8},{ }^{3} S_{1}^{8}\right]+q, & q \bar{q} \rightarrow \mathrm{Y}\left[{ }^{1} S_{0}^{8},{ }^{3} S_{1}^{8}\right]+g\end{array}$

calculate $p_{t}$ distribution - take $p_{t}>3 \mathrm{GeV} \rightarrow$ avoid soft gluon resummation $p_{\mathrm{Y}}=\left(\sqrt{M_{\mathrm{Y}}^{2}+p_{t}^{2}} \cosh (y), p_{t}, 0, \sqrt{M_{\mathrm{Y}}^{2}+p_{t}^{2}} \sinh (y)\right)$ 


\section{LD-Matrix-Elements \& BRs}

CS LDMEs from potential models via VRW (well-known)

- currently CO LDEMEs not known $\rightarrow$ large uncertainties if contribution sizable model dependence enters via $\mathcal{B}(\mathrm{Y}(6 S) \rightarrow \mathrm{Y}(n S) \pi \pi)$ estimate

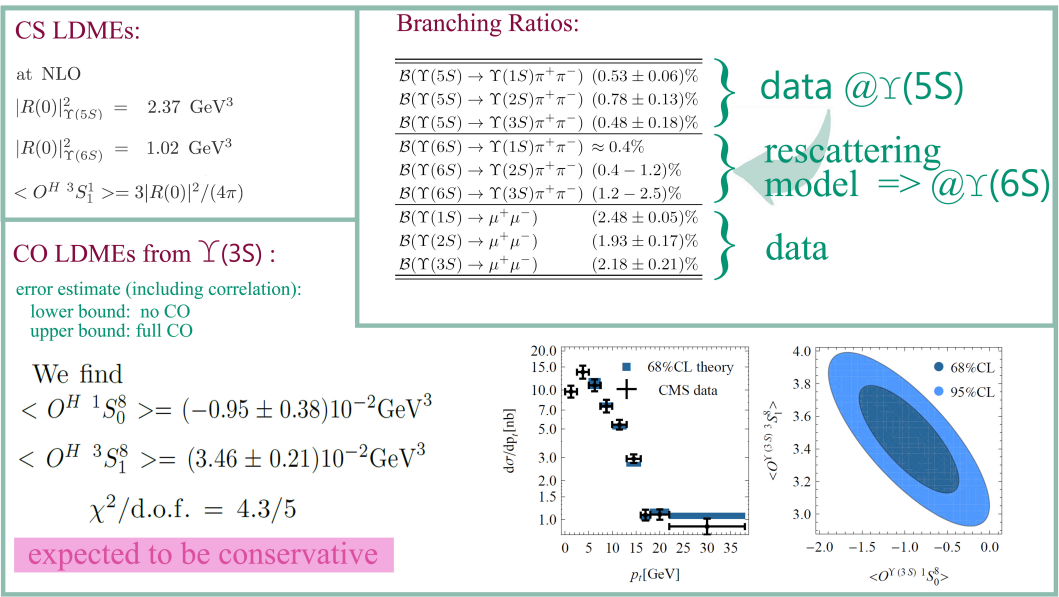




\section{$p \bar{p}(p) \rightarrow \mathrm{Y}(5 S, 6 S) \rightarrow\left(\mathrm{Y}(n S) \rightarrow \mu^{+} \mu^{-}\right) \pi^{+} \pi^{-}$in $\mathrm{pb}$}

\section{[Ali, Hambrock, Wang, PRD 13]}

\section{$\mathrm{LHCb}(7 \mathrm{TeV})$}

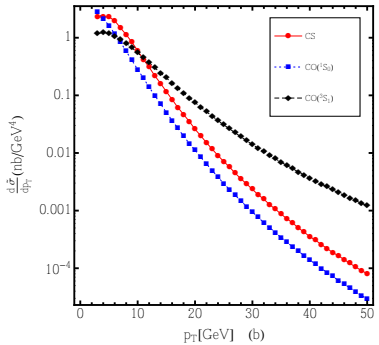

ATLAS \& CMS (7 TeV)

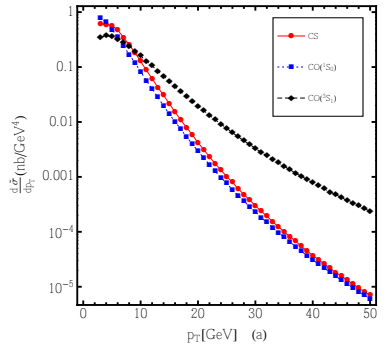

\begin{tabular}{lcccccc}
\hline \hline & & $\mathrm{Y}(5 S)$ & & & $\mathrm{Y}(6 S)$ & \\
& $n=1$ & $n=2$ & $n=3$ & $n=1$ & $n=2$ & $n=3$ \\
\hline Tevatron & {$[0.18,0.98]$} & {$[0.18,1.35]$} & {$[0.09,1.03]$} & {$[0.06,0.57]$} & {$[0.04,1.38]$} & {$[0.15,3.26]$} \\
LHC 7 & {$[0.86,5.26]$} & {$[0.86,6.74]$} & {$[0.44,5.56]$} & {$[0.29,3.13]$} & {$[0.21,7.57]$} & {$[0.72,17.9]$} \\
LHCb 7 & {$[0.20,1.48]$} & {$[0.20,1.89]$} & {$[0.10,1.56]$} & {$[0.07,0.89]$} & {$[0.05,2.16]$} & {$[0.17,5.13]$} \\
LHC 8 & {$[0.99,6.17]$} & {$[0.99,7.89]$} & {$[0.51,6.52]$} & {$[0.34,3.67]$} & {$[0.25,8.87]$} & {$[0.83,21.0]$} \\
LHCb 8 & {$[0.25,1.78]$} & {$[0.25,2.28]$} & {$[0.13,1.88]$} & {$[0.08,1.08]$} & {$[0.06,2.61]$} & {$[0.20,6.19]$} \\
LHC 14 & {$[1.79,11.7]$} & {$[1.79,14.9]$} & {$[0.92,12.3]$} & {$[0.61,7.02]$} & {$[0.45,17.0]$} & {$[1.50,40.2]$} \\
LHCb 14 & {$[0.52,3.70]$} & {$[0.52,4.74]$} & {$[0.27,3.91]$} & {$[0.18,2.25]$} & {$[0.13,5.43]$} & {$[0.43,12.9]$} \\
\hline \hline
\end{tabular}




\section{Search for $X_{b}$ decaying into $Y(1 S) \pi^{+} \pi^{-}$}

\section{[CMS Collaboration: BPH11016 (Kai Feng Chen)]}

- Exclusion limits as a function of the $X_{b}$ mass at 95\% C.L.

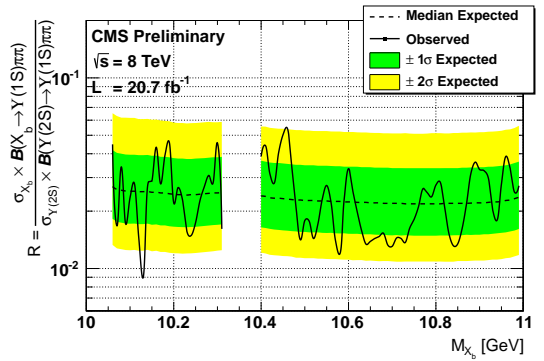

- For $X_{b}=Y_{b}(10876)$, the limit is: $\frac{\sigma\left(p p \rightarrow X_{b} \rightarrow Y(1 S) \pi^{+} \pi^{-}\right)}{\sigma\left(p p \rightarrow Y(2 S) \rightarrow Y(1 S) \pi^{+} \pi^{-}\right)}<0.02$,

- Using the CMS measurement $\sigma(p p \rightarrow \mathrm{Y}(2 S) X) \mathcal{B}\left(\mathrm{Y}(2 S) \rightarrow \mu^{+} \mu^{-}\right)=1.55 \mathrm{nb}$

we get $\sigma\left(p p \rightarrow X_{b} \rightarrow \mathrm{Y}(1 S) \pi^{+} \pi^{-}\right) \mathcal{B}\left(\mathrm{Y}(1 S) \rightarrow \mu^{+} \mu^{-}\right)<7.1 \mathrm{pb}$

- This is typically $\mathcal{O}(10)$ away from theoretical estimates 